\title{
THE STRAIN OF A GRAVITATING SPHERE OF VARIABLE DENSITY AND ELASTICITY*
}

BY

\section{M. HOSKINS}

\section{Introductory. Statement of the PROBLEM}

The problem of determining the strain of a gravitating elastic sphere from an initial condition of hydrostatic equilibrium, under the action of small disturbing forces having a spherical harmonic potential, has been solved for the case in which the density and elastic moduli are uniform throughout the body. It has also been solved for cases in which the density varies with the distance from the center, but with the restriction that the modulus of compression is infinite while the modulus of rigidity has a uniform value.

This problem is of especial interest because of its relation to estimates of the rigidity of the earth. Such estimates are based upon the comparison of the actual yielding of the earth to tidal and centrifugal forces (as inferred from certain refined observations) with the computed yielding of an elastic sphere having the same size and mass as the earth. For the purpose of such a comparison it is obviously desirable that the ideal sphere assumed in the computations should agree with the actual earth-sphere as closely as possible in all the elements essential to the problem. Now it is quite certain that the density of the earth varies greatly with distance from the center, that the material of the earth is far from incompressible, and that the earth as a whole is very much more rigid than the surface rocks. Our knowledge of the actual properties of the earth is much better represented by the assumption that all three of the quantities, density, modulus of compression, and modulus of rigidity are functions of distance from the center than by any of the more restricted assumptions which are essential to the above-mentioned solutions of the sphere problem. It is therefore desirable to solve, in as general a form as possible, the following problem:

A sphere of isotropic material, in which the density and elastic moduli are functions of distance from the center, and which would be in hydrostatic equilibrium under self-gravitation alone, is strained by small disturbing forces

* Presented to the Society, October 27, 1917 and April 5, 191؟. 
having a potential which is a spherical harmonic function of the coördinates and a simple harmonic function of the time; it is required to determine the strain.

The analysis given in the present paper leads to a solution of this problem which, though not fully general, is a considerable advance in generality over the restricted solutions above mentioned. The solution of the general problem is shown to depend upon that of a pair of simultaneous ordinary differential equations; and it is found that these may be solved in terms of convergent series when certain restrictions are imposed upon the functions expressing the density and elastic moduli. Moreover, these restrictions are not such as to detract seriously from the geodynamical interest of the solution.

The scope and order of the paper may be summarized as follows:

I. Introductory. Statement of the problem.

II. Establishment of fundamental equations in general form. The density and elastic moduli are here treated as unrestricted functions of distance from center, and the disturbing potential as a simple harmonic function of the time.

III. Solution of the equations when the density is variable while the elastic moduli are constants.* The density function is assumed to be rational and integral.

IV. Solution of equations when the density and both elastic moduli are variables. The assumption as to density is the same as in III, while the elastic moduli are represented by rational integral functions of a restricted form.

V. Particular solutions. Particular forms of the functions expressing density and elastic moduli are substituted in the formulas expressing the general solution given in IV, reducing these to the working formulas used in numerical computations.

VI. Application to the earth. Numerical results are given for the strain of a sphere having the size and mass of the earth, showing the effect of different assumptions regarding density, elasticity, and compressibility. Besides the results for the static problem, a series is also given for the case in which the disturbing potential is a simple harmonic function of the time.

\section{Establishment of the FUNDAMENTAL EQUATIONS}

1. Physical theory. The analys - which follows is a generalization of that used in a former paper dealing with a iass general form of the sphere problem, $\dagger$

* The assumptions of variable density and variable elasticity are found to be independent of each other as regards their effect on the fundamental equations, and it seems advantageous to present separately the two parts of the analysis leading to the most general form of the equations which express the solution.

† The strain of a gravitating, compressible elastic sphere, these $\mathrm{Tr}$ a $\mathrm{n}$ s a $\mathrm{ct}$ i o $\mathrm{n}$, vol. 11 (1910), pp. 203-248. 
but with an amendment to the physical theory. This amendment leads to certain changes in the fundamental equations; but though physically important, these changes are algebraically of minor effect, leaving the same general method of solution still applicable.*

The problem of the strain of an elastic solid from a condition of great initial stress cannot be treated by the equations of elastic deformation in the ordinary form, since these equations assume the proportionality of actual stress to strain. Some of the earlier papers on the strain of a gravitating sphere treated the problem as if the body were strained from an initial " natural" configuration in which gravitation did not act. Applied to a body of planetary dimensions such a solution leads to deformations far beyond the elastic limits of known materials; but even apart from this fact, the basal assumption can have no validity as applied to actual bodies. It is only on the assumption of incompressibility that this method of solution leads to valid results. If the material is incompressible the computed effect of gravitation alone is a condition of hydrostatic stress throughout the sphere, with zero strain; so that the determination by this method of the strain due to small disturbing forces combined with gravitation amounts to the same thing as the determination of the strain due to the disturbing forces alone from an initial condition of hydrostatic stress. $\dagger$ For a compressible material, however, the reasoning fails.

It is reasonable to suppose that a solid body is elastic for small deformations even if initially in a condition of great stress. It seems reasonable, also, to apply the ordinary laws of elasticity to such a case, with this change: the relation "stress is proportional to strain" is replaced by the relation "increment of stress is proportional to strain." $\ddagger$

* The defect in the physical theory as formerly stated was pointed out by the writer in a footnote to a later paper, these $\mathrm{Transactions,}$ vol. 11 (1910), p. 504. The same point was discussed by A. E. H. Love in his work Some problems of geodynamics (1911), in which the amended theory was applied in an able analysis of the sphere problem in the case of uniform density.

† This method was employed by G. Herglotz in a paper dealing with the strain of an incompressible sphere of variable density. Über die Elastizität der Erde bei Berücksichtigung ihrer variablen Dichte, Z e it s c hrift für $\mathbf{r}$ a the matik und Physik, vol. 52 (1905), p. 275. In this paper the solution for the general case of density varying with distance from the center is reduced to the solution of a linear ordinary differential equation of the sixth order whose coefficients depend upon the law of density, and the complete solution is given for certain cases of that law. When the assumption of incompressibility is introduced the analysis given in the present paper leads to a differential equation identical with that obtained by Herglotz.

¥ In the application of this principle in the writer's former paper cited above, "increment of stress" was computed by considering a definite volume-element before and during the strain. This was later seen to be an error, because the displacement of an element of matter causes a change in the gravitational force acting on that element which is of the same order of importance as the disturbing force. The reasonable method is rather to compare the stress-condi- 
The differential equations of strain, formed in accordance with this principle, involve the initial stresses; these are in general indeterminate, and the solution of the equations is therefore indeterminate unless something is assumed regarding the initial stress-condition. The assumption which has commonly been made, and which will be made in the analysis herein given, is that the initial condition is that of hydrostatic equilibrium. This assumption is not only especially simple but is especially interesting in the geodynamical problem, since in the case of a body of planetary dimensions like the earth the predominating stress throughout the greater part of the body is without doubt a normal pressure.*

2. Differential equations of strain of an isotropic elastic solid from a condition of initial stress. As a preliminary to the discussion of the problem of the sphere it is useful to establish the fundamental equations in as general a form as possible. We therefore consider first the general case of an isotropic elastic solid undergoing a small strain from any state of initial stress; the fundamental assumption being that increment of stress is connected with strain by the same relations which, in the ordinary theory, are assumed to connect actual stress with strain.

In establishing the differential equations it is convenient to use reciangular coördinates; polar coördinates are however employed in the application to the sphere $\dagger$ The notation for displacements, strains, and stresses will for the most part be that adopted in A. E. H. Love's treatise The Mathematical Theory of Elasticity, second edition; axial components of displacement are, however, denoted by $u_{x}, u_{y}, u_{z}$ instead of $u, v, w$.

The equations of motion for an element of the body are

$$
\begin{gathered}
\rho \frac{\partial^{2} u_{x}}{\partial t^{2}}=\rho X+\frac{\partial X_{x}}{\partial x}+\frac{\partial X_{y}}{\partial y}+\frac{\partial X_{z}}{\partial z} \\
\rho \frac{\partial^{2} u}{\partial t^{2}}=\rho Y+\frac{\partial Y_{x}}{\partial x}+\frac{\partial Y_{y}}{\partial y}+\frac{\partial Y_{z}}{\partial z} \\
\rho \frac{\partial^{2} u_{z}}{\partial t^{2}}=\rho Z+\frac{\partial Z_{x}}{\partial x}+\frac{\partial Z_{y}}{\partial y}+\frac{\partial Z_{z}}{\partial z}
\end{gathered}
$$

These equations are independent of elastic theory.

tion of an individual element of matter before and during strain. This is the amendment to the physical theory referred to above. The defective theory had also been employed in $\mathrm{a}$ paper by A. E. H. Love, The graintational stability of the earth, P h i l o s o p h i c a l T r a n sactions of the Royal Societ y, London, A, vol. 207 (1907), p. 171.

* This method of treating the geodynamical problem seems to have been first suggested by Lord Rayleigh. See paper On the dilatational stability of the earth, P r oc e e d i n $\mathbf{g}$ of the Roy a l S o c i e ty, London, A, vol. 77 (1906).

$\dagger$ Attention is called to the fact that after the change to polar coördinates the letters $x$, $y, z$ are given new meanings. 
The ordinary stress-strain relations for an isotropic elastic solid are expressed by the six equations

$$
\begin{gathered}
X_{x}=\lambda \Delta+2 \mu \frac{\partial u_{x}}{\partial x}, \quad Y_{y}=\lambda \Delta+2 \mu \frac{\partial u_{y}}{\partial y}, \quad Z_{z}=\lambda \Delta+2 \mu \frac{\partial u_{z}}{\partial z} \\
Y_{z}=Z_{y}=\mu\left(\frac{\partial u_{z}}{\partial y}+\frac{\partial u_{y}}{\partial z}\right), \quad Z_{x}=X_{z}=\mu\left(\frac{\partial u_{x}}{\partial z}+\frac{\partial u_{z}}{\partial x}\right) \\
X_{y}=Y_{x}=\mu\left(\frac{\partial u_{y}}{\partial x}+\frac{\partial u_{x}}{\partial y}\right) .
\end{gathered}
$$

Equations (2) apply to the case in which the body is strained from its " natural "state, i.e., from a condition of zero stress.

Let it now be supposed that the body is initially in equilibrium under the action of any bodily and surface forces, so that initial stresses exist throughout the material, and that $u_{x}, u_{y}, u_{z}$ denote displacement from this initial configuration; it will be assumed that equations like (2) hold with the substitution for each stress-component of the increment of that stress-component due to the strain.

Let $\delta X_{x}, \delta Y_{y}, \cdots$ denote these increments of the stress-components for an element of material initially at $(x, y, z)$; then the assumption is expressed by the equations

$$
\begin{gathered}
\delta X_{x}=\lambda \Delta+2 \mu \frac{\partial u_{x}}{\partial x}, \quad \delta Y_{y}=\lambda \Delta+2 \mu \frac{\partial u_{y}}{\partial y}, \quad \delta Z_{z}=\lambda \Delta+2 \mu \frac{\partial u_{z}}{\partial z} \\
\delta Y_{z}=\delta Z_{y}=\mu\left(\frac{\partial u_{z}}{\partial y}+\frac{\partial u_{y}}{\partial z}\right), \quad \delta Z_{x}=\delta X_{z}=\mu\left(\frac{\partial u_{x}}{\partial z}+\frac{\partial u_{z}}{\partial x}\right) \\
\delta X_{y}=\delta Y_{x}=\mu\left(\frac{\partial u_{y}}{\partial x}+\frac{\partial u_{x}}{\partial y}\right)
\end{gathered}
$$

These equations give, by differentiation and addition,

$$
\begin{aligned}
\frac{\partial}{\partial x} \delta X_{x}+\frac{\partial}{\partial y} \delta & X_{y}+\frac{\partial}{\partial z} \delta X_{z}=(\lambda+\mu) \frac{\partial \Delta}{\partial x}+\mu \nabla^{2} u_{x} \\
+ & \frac{\partial \lambda}{\partial x} \Delta+2 \frac{\partial \mu}{\partial x} \frac{\partial u_{x}}{\partial x}+\frac{\partial \mu}{\partial y}\left(\frac{\partial u_{y}}{\partial x}+\frac{\partial u_{x}}{\partial y}\right)+\frac{\partial \mu}{\partial z}\left(\frac{\partial u_{x}}{\partial z}+\frac{\partial u_{z}}{\partial x}\right)
\end{aligned}
$$

with two similar equations which may be written by cyclic permutation of $x, y, z$.

Let the components of actual stress at the point $(x, y, z)$ before strain be $X_{x}, X_{y}, \cdots$; and let $X_{x}^{\prime}, X_{y}^{\prime}, \cdots$ denote the same quantities at the same point during strain. Then the increment of stress for an individual element of 
material initially at $(x, y, z)$ is given by equations like the following:

$$
\delta X_{x}=X_{x}^{\prime}-X_{x}+u_{x} \frac{\partial X_{x}}{\partial x}+u_{y} \frac{\partial X_{x}}{\partial y}+u_{z} \frac{\partial X_{x}}{\partial z}
$$

and these values substituted in (4) reduce the first member to the expression

$$
\begin{aligned}
& \left(\frac{\partial X_{x}^{\prime}}{\partial x}+\frac{\partial X_{y}^{\prime}}{\partial y}+\frac{\partial X_{z}^{\prime}}{\partial z}\right)-\left(\frac{\partial X_{x}}{\partial x}+\frac{\partial X_{y}}{\partial y}+\frac{\partial X_{z}}{\partial z}\right) \\
& +\frac{\partial}{\partial x}\left(u_{x} \frac{\partial X_{x}}{\partial x}+u_{y} \frac{\partial X_{x}}{\partial y}+u_{z} \frac{\partial X_{x}}{\partial z}\right)+\frac{\partial}{\partial y}\left(u_{x} \frac{\partial X_{y}}{\partial x}+u_{y} \frac{\partial X_{y}}{\partial y}+u_{z} \frac{\partial X_{y}}{\partial z}\right) \\
& \quad+\frac{\partial}{\partial z}\left(u_{x} \frac{\partial X_{z}}{\partial x}+u_{y} \frac{\partial X_{z}}{\partial y}+u_{z} \frac{\partial X_{z}}{\partial z}\right) .
\end{aligned}
$$

But the first of equations (1) gives

$$
\begin{aligned}
& \frac{\partial X_{x}}{\partial x}+\frac{\partial X_{\ddot{\prime}}}{\partial y}+\frac{\partial X_{z}}{\partial z}=-\rho X, \\
& \frac{\partial X_{x}^{\prime}}{\partial x}+\frac{\partial X_{y}^{\prime}}{\partial y}+\frac{\partial X_{z}^{\prime}}{\partial z}=-\rho^{\prime} X^{\prime}+\rho^{\prime} \frac{\partial^{2} u_{x}}{\partial t^{2}},
\end{aligned}
$$

in which accented letters denote values during strain and unaccented letters corresponding values before strain at the same point $(x, y, z)$. Also

$$
\rho^{\prime}=\rho(1-\Delta)-\left(u_{x} \frac{\partial \rho}{\partial x}+u_{y} \frac{\partial \rho}{\partial y}+u_{z} \frac{\partial \rho}{\partial z}\right)
$$

and since $\rho, X$ may replace $\rho^{\prime}, X^{\prime}$ in terms containing $u_{x}, u_{y}$, or $u_{z}$, the expression (6) becomes

$$
\begin{array}{r}
\rho\left(\frac{\partial^{2} u_{x}}{\partial t^{2}}+X-X^{\prime}+X \Delta\right)+X\left(u_{x} \frac{\partial \rho}{\partial x}+u_{y} \frac{\partial \rho}{\partial y}+u_{z} \frac{\partial \rho}{\partial z}\right) \\
+\frac{\partial}{\partial x}\left(u_{x} \frac{\partial X_{x}}{\partial x}+u_{y} \frac{\partial X_{x}}{\partial y}+u_{z} \frac{\partial X_{x}}{\partial z}\right)+\frac{\partial}{\partial y}\left(u_{x} \frac{\partial X_{y}}{\partial x}+u_{y} \frac{\partial X_{y}}{\partial y}+u_{z} \frac{\partial X_{y}}{\partial z}\right) \\
+\frac{\partial}{\partial z}\left(u_{x} \frac{\partial X_{z}}{\partial x}+u_{y} \frac{\partial X_{z}}{\partial y}+u_{z} \frac{\partial X_{z}}{\partial z}\right) .
\end{array}
$$

Equation (4), with (9) substituted for the first member, is one of three differential equations applicable to the general case of strain of an isotropic elastic solid from a condition of initial stress. The six stress-components whose derivatives appear in these equations are connected by the relations obtained from (1) (with the acceleration terms omitted); but the solution will be indeterminate unless something more is known concerning the initial stresses. 
3. Simplification of equations when initial stress is hydrostatic. It will hereafter be assumed that the initial stress-condition is that of hydrostatic equilibrium, so that all terms involving tangential stress-components vanish, while $X_{x}=Y_{y}=Z_{z}$. Using (1), the expression (9) may now be written

$$
\begin{aligned}
\rho\left(\frac{\partial^{2} u_{x}}{\partial t^{2}}+X-X^{\prime}+X \Delta\right)+X\left(u_{x} \frac{\partial \rho}{\partial x}+u_{y} \frac{\partial \rho}{\partial y}+u_{z} \frac{\partial \rho}{\partial z}\right) \\
-\frac{\partial}{\partial x}\left(\rho u_{x} X+\rho u_{y} Y+\rho u_{z} Z\right) .
\end{aligned}
$$

4. Form assumed by equations when the body is a gravitating sphere. Let it now be assumed that the body is a sphere, and that $\rho, \lambda$, and $\mu$ vary only with $r$, the distance from the center. If $V$ denotes the gravitation potential before strain (a function of $r$ only),

$$
X=\frac{x}{r} \frac{d V}{d r}, \quad Y=\frac{y}{r} \frac{d V}{d r}, \quad Z=\frac{z}{r} \frac{d V}{d r}
$$

Hence if $u_{r}$ denotes radial displacement,

Also

$$
\begin{aligned}
u_{x} X+u_{y} Y+u_{z} Z & =u_{r} \frac{d V}{d r} ; \\
\frac{\partial}{\partial x}\left(\rho u_{x} X+\rho u_{y} Y+\rho u_{z} Z\right) & =\frac{\partial}{\partial x}\left(\rho u_{r} \frac{d V}{d r}\right) \\
& =\rho \frac{\partial}{\partial x}\left(u_{r} \frac{d V}{d r}\right)+u_{r} \frac{x}{r} \frac{d \rho}{d r} \frac{d V}{d r} .
\end{aligned}
$$

$$
X\left(u_{x} \frac{\partial \rho}{\partial x}+u_{y} \frac{\partial \rho}{\partial y}+u_{z} \frac{\partial \rho}{\partial z}\right)=u_{r} \frac{x}{r} \frac{d \rho}{d r} \frac{d V}{d r}
$$

Assuming now that the bodily forces during strain are derived from a potential $V^{\prime}$, the expression (10) reduces to the following:

$$
\rho\left[\frac{\partial^{2} u_{x}}{\partial t^{2}}+\frac{\partial V}{\partial x} \Delta-\frac{\partial}{\partial x}\left(V^{\prime}-V+u_{r} \frac{d V}{d r}\right)\right] \text {. }
$$

Substituting this for the first member of (4), and writing $E_{x}$ for the terms in the second member which contain derivatives of $\lambda$ and $\mu$, we obtain the following as one of the three differential equations for the strain of a gravitating sphere: 


$$
\begin{aligned}
(\lambda+\mu) \frac{\partial \Delta}{\partial x}+\mu \nabla^{2} u_{x} & +E_{x} \\
& =\rho\left[\frac{\partial^{2} u_{x}}{\partial t^{2}}+\frac{\partial V}{\partial x} \Delta-\frac{\partial}{\partial x}\left(V^{\prime}-V+u_{r} \frac{d V}{d r}\right)\right] .
\end{aligned}
$$

The other two equations are obtained by cyclic permutation of $x, y, z$.

The first members of these equations are the same as would be obtained if there were no initial stress. The equations are in fact identical with those which would apply to strain from zero-stress under the action of forces whose axial components (per unit mass) are

$$
\begin{gathered}
\frac{\partial}{\partial x}\left(V^{\prime}-V+u_{r} \frac{d V}{d r}\right)-\frac{\partial V}{\partial x} \Delta, \quad \frac{\partial}{\partial y}\left(V^{\prime}-V+u_{r} \frac{d V}{d r}\right)-\frac{\partial V}{\partial y} \Delta, \\
\frac{\partial}{\partial z}\left(V^{\prime}-V+u_{r} \frac{d V}{\partial r}\right)-\frac{\partial V}{d z} \Delta .
\end{gathered}
$$

In other words the strain may be treated as if due to three sets of bodily forces:

(a) Forces having potential $V^{\prime}-V$; these include the disturbing force and the change in the gravitational force due to the changed configuration of the attracting mass.

(b) Forces having potential $u_{r}(d V / d r)$.

(c) The increment of gravitational force per unit volume due to the change $-\rho \Delta$ in the density of the attracted element.

5. Equations in polar coördinates for gravitating sphere. We now pass to polar coördinates, replacing $x, y, z$ by $r, \theta, \phi$ as independent variables, and $u_{x}, u_{y}, u_{z}$ by $u_{r}, u_{\theta}, u_{\phi}$ as component displacements, according to the following scheme of direction cosines.

\begin{tabular}{|c|c|c|c|}
\hline & $r$ & $\theta$ & $\phi$ \\
\hline$x$ & $\sin \theta \cos \phi$ & $\cos \theta \cos \phi$ & $-\sin \phi$ \\
\hline$y$ & $\sin \theta \sin \phi$ & $\cos \theta \sin \phi$ & $\cos \phi$ \\
\hline$z$ & $\cos \theta$ & $-\sin \theta$ & 0 \\
\hline
\end{tabular}

Each of the required equations is obtained by multiplying the three equations like (16) by the proper direction cosines and adding. The second members of the resulting equations may be written by inspection (noticing that, to the first order of small quantities, the acceleration components are $\left.\partial^{2} u_{r} / \partial t^{2}, \partial^{2} u_{\theta} / \partial t^{2}, \partial^{2} u_{\phi} / \partial t^{2}\right)$. The first members, aside from the terms obtained from $E_{x}, E_{y}, E_{z}$, are given in treatises dealing with the case in which $\lambda$ and $\mu$ are constants. * Let the terms involving derivatives of $\lambda$ and $\mu$

* See A. E. H. Love's Treatise on the Mathematical Theory of Elasticity, second edition (1906), p. 138. For the values of $\varpi_{r}, \varpi_{\theta}, \varpi_{\phi}, \Delta$, see p. 56 . 
be $E_{r}, E_{0}, E_{\phi}$; then the three equations in polar coördinates are

$$
\begin{aligned}
& (\lambda+2 \mu) \frac{\partial \Delta}{\partial r}-\frac{2 \mu}{r \sin \theta}\left[\frac{\partial}{\partial \theta}\left(\varpi_{\phi} \sin \theta\right)-\frac{\partial \varpi_{\theta}}{\partial \phi}\right]+E_{r} \\
& =\rho\left[\frac{\partial^{2} u_{r}}{\partial t^{2}}+\frac{\partial V}{\partial r} \Delta-\frac{\partial}{\partial r}\left(V^{\prime}-V+u_{r} \frac{d V}{d r}\right)\right], \\
& \frac{\lambda+2 \mu}{r} \frac{\partial \Delta}{\partial \theta}-\frac{2 \mu}{r \sin \theta}\left[\frac{\partial \varpi_{r}}{\partial \phi}-\frac{\partial}{\partial r}\left(r \varpi_{\phi} \sin \theta\right)\right]+E_{\theta} \\
& =\rho\left[\frac{\partial^{2} u_{\theta}}{\partial t^{2}}-\frac{1}{r} \frac{\partial}{\partial \theta}\left(V^{\prime}-V+u_{r} \frac{d V}{d r}\right)\right], \\
& \frac{\lambda+2 \mu}{r \sin \theta} \frac{\partial \Delta}{\partial \phi}-\frac{2 \mu}{r}\left[\frac{\partial}{\partial r}\left(r \varpi_{\theta}\right)-\frac{\partial \varpi_{r}}{\partial \theta}\right]+E_{\phi} \\
& =\rho\left[\frac{\partial^{2} u_{\phi}}{\partial t^{2}}-\frac{1}{r \sin \theta} \frac{\partial}{\partial \phi}\left(V^{\prime}-V+u_{r} \frac{d V}{d r}\right)\right] \text {. }
\end{aligned}
$$

Here $\varpi_{r}, \varpi_{\theta}, \varpi_{\phi}$ are rotational components of strain.

It remains to determine $E_{r}, E_{0}, E_{\phi}$ by carrying out the process above outlined. If $\lambda$ and $\mu$ are unrestricted functions of the coördinates, the resulting values are

$$
\begin{aligned}
E_{r}=\Delta \frac{\partial \lambda}{\partial r}+2 \frac{\partial \mu}{\partial r} \frac{\partial u_{r}}{\partial r}+\frac{1}{r} \frac{\partial \mu}{\partial \theta}\left(\frac{1}{r} \frac{\partial u_{r}}{\partial \theta}+\frac{\partial u_{\theta}}{\partial r}-\frac{u_{\theta}}{r}\right) & \\
& +\frac{1}{r \sin \theta} \frac{\partial \mu}{\partial \phi}\left(\frac{1}{r \sin \theta} \frac{\partial u_{r}}{\partial \phi}+\frac{\partial u_{\phi}}{\partial r}-\frac{u_{\phi}}{r}\right) \\
E_{\theta}=\frac{\Delta}{r} \frac{\partial \lambda}{\partial \theta}+\frac{\partial \mu}{\partial r}\left(\frac{1}{r} \frac{\partial u_{r}}{\partial \theta}+\frac{\partial u_{\theta}}{\partial r}-\frac{u_{\theta}}{r}\right)+\frac{2}{r} \frac{\partial \mu}{\partial \theta}\left(\frac{u_{r}}{r}+\frac{1}{r} \frac{\partial u_{\theta}}{\partial \theta}\right) & +\frac{1}{r \sin \theta} \frac{\partial \mu}{\partial \phi}\left(\frac{1}{r \sin \theta} \frac{\partial u_{\theta}}{\partial \phi}+\frac{1}{r} \frac{\partial u_{\phi}}{\partial \theta}-\frac{u_{\phi}}{r} \cot \theta\right) \\
E_{\phi}=\frac{\Delta}{r \sin \theta} \frac{\partial \lambda}{\partial \phi}+\frac{\partial \mu}{\partial r}( & \left.\frac{1}{r \sin \theta} \frac{\partial u_{r}}{\partial \phi}+\frac{\partial u_{\phi}}{\partial r}-\frac{u_{\phi}}{r}\right) \\
& +\frac{1}{r} \frac{\partial \mu}{\partial \theta}\left(\frac{1}{r \sin \theta} \frac{\partial u_{\theta}}{\partial \phi}+\frac{1}{r} \frac{\partial u_{\phi}}{\partial \theta}-\frac{u_{\phi}}{r} \cot \theta\right) \\
& +\frac{2}{r \sin \theta} \cdot \frac{\partial \mu}{\partial \phi}\left(\frac{u_{r}}{r}+\frac{u_{\theta}}{r} \cot \theta+\frac{1}{r \sin \theta} \frac{\partial u_{\phi}}{\partial \phi}\right) .
\end{aligned}
$$

Since in the subsequent applications of equations (18) it is to be assumed that $\lambda$ and $\mu$ are functions of $r$ only, the expressions (19) are much simplified.*

* Considerable algebraic work is involved in the deduction of (19), but it is merely the routine work of transformation of coördinates and is therefore omitted. Although the application in the present paper is limited to the case in which $\lambda$ and $\mu$ are independent of $\theta$ and $\phi$, it seems desirable to record the general expressions. 
6. The boundary conditions. The condition which will be assumed at the boundary surface is that the increment of stress on this surface is zero.* Making the same assumptions as in equations (3), but using polar coördinates, the increments of normal and tangential stress on a surface initially perpendicular to the radius vector are

$$
\begin{aligned}
& \delta_{\overline{r r}}=\lambda \Delta+2 \mu \frac{\partial u_{r}}{\partial r}, \\
& \delta_{\overline{r \theta}}=\mu\left(\frac{\partial u_{\theta}}{\partial r}-\frac{u_{\theta}}{r}+\frac{1}{r} \frac{\partial u_{r}}{\partial \theta}\right), \\
& \delta_{\tilde{r}_{\bar{\phi}}}=\mu\left(\frac{\partial u_{\phi}}{r \partial}-\frac{u_{\phi}}{r}+\frac{1}{r \sin \theta} \frac{\partial u_{r}}{\partial \phi}\right) ;
\end{aligned}
$$

and these are to vanish when $r=a$.

7. Case in which the disturbing potential is a spherical harmonic function of the coördinates and a simple harmonic function of the time. We now assume that the disturbing potential is proportional to $S_{i} r^{i}$, where $S_{i}$ is a spherical surface harmonic' of order $i$ and a simple harmonic function of the time. In this case equations (18) may be reduced to simultaneous ordinary differential equations by the following method. $\dagger$

Assume

$$
u_{r}=u S_{i}, \quad u_{\theta}=v \frac{\partial S_{i}}{\partial \theta}, \quad u_{\phi}=\frac{v}{\sin \theta} \frac{\partial S_{i}}{\partial \phi},
$$

in which $u, v$ are functions of $r$ only. Substituting in the known formulas for $\Delta, \varpi_{r}, \varpi_{0}, \varpi_{\phi}$, making use of the partial differential equation

$$
\frac{1}{\sin ^{2} \theta} \frac{\partial^{2} S_{i}}{\partial \phi^{2}}+\frac{1}{\sin \theta} \frac{\partial}{\partial \theta}\left(\sin \theta \frac{\partial S_{i}}{\partial \theta}\right)+i(i+1) S_{i}=0,
$$

and introducing the notation

$$
y=\frac{1}{r^{2}} \frac{d\left(r^{2} u\right)}{d r}-i(i+1) \frac{v}{r}, \quad z=\frac{1}{r}\left(\frac{d(r v)}{d r}-u\right),
$$

we find

$$
\Delta=y S_{i}, \quad 2 \varpi_{r}=0, \quad 2 \varpi_{\theta}=-\frac{z}{\sin \theta} \frac{\partial S_{i}}{\partial \phi}, \quad 2 \varpi_{\phi}=z \frac{\partial S_{i}}{\partial \theta} ;
$$

so that the first members of (18), exclusive of the terms $E_{r}, E_{\theta}, E_{\phi}$, take

* The amendment to the physical theory, referred to above, affects the boundary conditions as well as the differential equations. The increment of stress is zero, not for the fixed surface $r=a$, but for the material surface initially at $r=a$.

t This method was used in the writer's previous papers dealing with less general cases of the sphere problem. Some of the details given in those papers are here omitted. See these T r a n s a c tio n s, vol. 11 (1910), p. 238 et seq.; also p. 495 et seq. 
the forms

$$
\left[(\lambda+2 \mu) \frac{d y}{d r}+i(i+1) \mu \frac{z}{r}\right] S_{i}, \quad\left[(\lambda+2 \mu) \frac{y}{r}+\frac{\mu}{r} \frac{d(r z)}{d r}\right] \frac{\partial S_{i}}{\partial \theta},
$$

while from (19),

$$
\left[(\lambda+2 \mu) \frac{y}{r}+\frac{\mu}{r} \frac{d(r z)}{d r}\right] \frac{1}{\sin \theta} \frac{\partial S_{i}}{\partial \phi} \text {; }
$$

$$
\begin{gathered}
E_{r}=\left(\frac{d \lambda}{d r} y+2 \frac{d \mu}{d r} \frac{d u}{d r}\right) S_{i}, \quad E_{\theta}=\frac{d \mu}{d r}\left(\frac{u}{r}+\frac{d v}{d r}-\frac{v}{r}\right) \frac{\partial S_{i}}{\partial \theta}, \\
E_{\phi}=\frac{d \mu}{d r}\left(\frac{u}{r}+\frac{d v}{d r}-\frac{v}{r}\right) \frac{1}{\sin \theta} \frac{\partial S_{i}}{\partial \phi} .
\end{gathered}
$$

Again, since $S_{i}$ is a simple harmonic function of $t$, we may put

$$
\frac{\partial^{2} S_{i}}{\partial t^{2}}=-p^{2} S_{i}
$$

in which $p$ is a constant. Hence the acceleration components in (18) become

$$
\frac{\partial^{2} u_{r}}{\partial t^{2}}=-p^{2} u S_{i}, \quad \frac{\partial^{2} u_{\theta}}{\partial t^{2}}=-p^{2} v \frac{\partial S_{i}}{\partial \theta}, \quad \frac{\partial^{2} u_{\phi}}{\partial t^{2}}=-\frac{p^{2} v}{\sin \theta} \frac{\partial S_{i}}{\partial \phi}
$$

It remains to express the values of $V$ and $V^{\prime}$ for substitution in the second members of (18).

Let the potential of the disturbing forces be

$$
W=\frac{c g}{2 a^{i-1}} r^{i} S_{i}
$$

$c$ being a constant which measures the intensity of the disturbing force, and $g$ the surface value of the gravitational attraction per unit mass.

If $\rho_{m}$ is the mean density of the sphere and $a$ the radius,

$$
V=\frac{3 g}{\rho_{m} a}\left[\begin{array}{l}
1 \\
r
\end{array} \int_{0}^{r} \rho r^{2} d r+\int_{r}^{a} \rho r d r\right]
$$

The potential $V^{\prime}$ is made up of $W$ and the gravitation potential of the strained body; hence if $U$ denotes the increment of the gravitation potential caused by the strain,

$$
V^{\prime}-V=W+U
$$

The value of $U$ may be expressed as the sum of two parts, one of which is the potential of a distribution of matter of density

$$
-\rho \Delta-u_{r} \frac{d \rho}{d r}
$$


throughout the sphere, the other that of a surface layer of thickness equal to the surface value of $u_{r}$. Since, by virtue of (21) and (24),

$$
-\rho \Delta-u_{r} \frac{d \rho}{d r}=-\left(\rho y+u \frac{d \rho}{d r}\right) S_{i},
$$

the former of the two parts of $U$ is

$$
-\frac{4 \pi \gamma S_{i}}{2 i+1}\left[\frac{1}{r^{i+1}} \int_{0}^{r}\left(\rho y+u \frac{d \rho}{d r}\right) r^{i+2} d r+r^{i} \int_{r}^{a}\left(\rho y+u \frac{d \rho}{d r}\right) \frac{d r}{r^{i-1}}\right]
$$

while the latter is

$$
\frac{4 \pi \gamma S_{i}}{2 i+1} \rho_{1} u_{1} a\left(\frac{r}{a}\right)^{i}
$$

in which the suffix $(1)$ refers to surface values. Combining (33) and (34) and integrating by parts the terms containing $d \rho / d r$, we find

$$
\begin{aligned}
U=-\frac{3 g S_{i}}{(2 i+1) \rho_{m} a}\left[\frac { 1 } { r ^ { i + 1 } } \int _ { 0 } ^ { r } \rho \left(y r^{i+2}\right.\right. & \left.-\frac{d}{d r}\left(u r^{i+2}\right)\right) d r \\
& \left.+r^{i} \int_{r}^{a} \rho\left(\frac{y}{r^{i-1}}-\frac{d}{d r}\left(\frac{u}{r^{i-1}}\right)\right) d r\right],
\end{aligned}
$$

in which $4 \pi \gamma$ has been replaced by $3 g / \rho_{m} a$. The value of $V^{\prime}-V$ is obtained by combining (29) and (35), in accordance with (31).

From (21), (24), and (30),

$$
u_{r} \frac{d V}{d r}=-\frac{3 g u S_{i}}{\rho_{m} a r^{2}} \int_{0}^{r} \rho r^{2} d r, \quad \Delta \frac{d V}{d r}=-\frac{3 g y S_{i}}{\rho_{m} a r^{2}} \int_{0}^{r} \rho r^{2} d r .
$$

The final result of the substitution of (21) in (18) is obtained by combining the foregoing partial results. The first members of the resulting equations are made up of (25) and (26), while the second members are obtained from (28), (29), (35), and (36). After cancellation of the factors $S_{i}, \partial S_{i} / \partial \theta$, and $(1 / \sin \theta)\left(\partial S_{i} / \partial \phi\right)$, the three equations are independent of $\theta, \phi$, and $t$, the second and third equations being in fact identical. The solution of the system (18) is thus reduced to that of two simultaneous ordinary differential equations with $r$ as independent variable and $u, v$ as the functions to be determined. These two equations, after multiplication by $r$, may be written as follows:

$$
\begin{aligned}
&(\lambda+2 \mu) r \frac{d y}{d r}+i(i+1) \mu z+ r \frac{d \lambda}{d r} y+2 r \frac{d \mu}{d r} \frac{d u}{d r} \\
&=\frac{g \rho a}{\rho_{m}}\left(r \frac{d R}{d r}-\frac{3 y}{a^{2} r} \int_{0}^{r} \rho r^{2} d r\right)-\rho p^{2} r u \\
&(\lambda+2 \mu) y+\mu \frac{d(r z)}{d r}+r \frac{d \mu}{d r}\left(\frac{u}{r}+\frac{d v}{d r}-\frac{v}{r}\right)=\frac{g \rho a}{\rho_{m}} R-\rho p^{2} r v
\end{aligned}
$$




$$
\begin{aligned}
R= & \frac{\rho_{m}}{g a S_{i}}\left(V-V^{\prime}-u_{r} \frac{d V}{d r}\right) \\
= & \frac{3}{(2 i+1) a^{2}}\left[\frac{1}{r^{i+1}} \int_{0}^{r} \rho\left(y r^{i+2}-\frac{d}{d r}\left(u r^{i+2}\right)\right) d r\right. \\
& \left.\quad+r^{i} \int_{r}^{a} \rho\left(\frac{y}{r^{i-1}}-\frac{d}{d r}\left(\frac{u}{r^{i-1}}\right)\right) d r\right]+\frac{3 u}{a^{2} r^{2}} \int_{0}^{r} \rho r^{2} d r-\frac{c \rho_{m}}{2}\left(\frac{r}{a}\right)^{i} .
\end{aligned}
$$

The substitution of $(21)$ in (20) reduces the boundary conditions to the two equations

$$
\lambda y+2 \mu \frac{d u}{d r}=0, \quad \mu\left(\frac{d v}{d r}-\frac{v}{r}+\frac{u}{r}\right)=0,
$$

to be satisfied when $r=a$.

It will be shown that the solution of (37) subject to the conditions (39) may be obtained in the form of infinite convergent series in a comprehensive class of cases in which $\rho, \lambda$, and $\mu$ are variable. The solution will first be given on the assumption that $\lambda$ and $\mu$ are constants while $\rho$ is expressible as a rational integral function of $r$. It will then be shown that the effect of assuming $\lambda$ and $\mu$ also to be rational integral functions of $r$ is merely to add terms to the main equations employed in the solution, and that when these functions are restricted in a certain way the series expressing $u$ and $v$ are still convergent.

III. Solution of EQUATIONS WHEN THE DENSITY FUNCTION IS RATIONAL AND INTEGRAL AND THE ELASTIC MODULI ARE CONSTANTS

1. Solution when both elastic moduli are finite. Assuming $\lambda$ and $\mu$ to be constants, let

$$
\rho=\rho_{0}\left(1+k_{1} x+k_{2} x^{2}+\cdots\right),
$$

in which $\rho_{0}, k_{1}, k_{2}, \cdots$ are constants, and

$$
x=\frac{r}{a} .
$$

Hereafter $x$ will replace $r$ as independent variable, and $u, v$ will be replaced by $e, \alpha$, defined by the equations*

$$
e=\frac{u}{r}, \quad \alpha=\frac{v}{r} .
$$

Also, since $p^{2} a$ is of the same dimensions as acceleration, let

$$
p^{2} a=n g \text {. }
$$

* It will later appear that, in the important case $i=2, e$ and $\alpha$ have simple physical meanings. It is seen that $x, e$, and $\alpha$ (as well as $y$ and $z$ already defined) are abstract numbers. 
Equations (37) and (38) now take the following forms:

$$
(\lambda+2 \mu) x \frac{d y}{d x}+i(i+1) \mu z=\frac{g \rho a}{\rho_{m}}\left(x \frac{d R}{d x}-\frac{3 y}{x} \int_{0}^{x} \rho x^{2} d x-n \rho_{m} e x^{2}\right),
$$

$$
\begin{gathered}
(\lambda+2 \mu) y+\mu \frac{d(x z)}{d x}=\frac{g \rho a}{\rho_{m}}\left(R-n \rho_{m} \alpha x^{2}\right) \\
R=\frac{3}{2 i+1}\left[\frac{1}{x^{i+1}} \int_{0}^{x} \rho\left(y x^{i+2}-\frac{d}{d x}\left(e x^{i+3}\right)\right) d x\right. \\
\left.+x^{i} \int_{x}^{1} \rho\left(\frac{y}{x^{i-1}}-\frac{d}{d x}\left(\frac{e}{x^{i-2}}\right)\right) d x\right]+\frac{3 e}{x} \int_{0}^{x} \rho x^{2} d x-\frac{c \rho_{m}}{2} x^{i}
\end{gathered}
$$

while the boundary conditions (39) become

$$
\lambda y+2 \mu \frac{d(e x)}{d x}=0, \quad \mu\left(x \frac{d \alpha}{d x}+e\right)=0, \quad(\text { when } x=1) .
$$

If we now assume

$$
\begin{array}{cl}
y=\sum_{m=i}^{\infty} C_{m} x^{m}, & z=\sum_{m=i}^{\infty} D_{m} x^{m}, \\
e=\sum_{m=i-2}^{\infty} A_{m} x^{m}, & \alpha=\sum_{m=i-2}^{\infty} B_{m} x^{m},
\end{array}
$$

it is found that the coefficients $C_{m}, D_{m}, A_{m}, B_{m}$ may be so determined as to satisfy identically both (44) and (46). The solution may proceed as follows.

Notice first that $A_{m}$ and $B_{m}$ may be expressed in terms of $C_{m}$ and $D_{m}$ by means of (23), which may now be written

$$
y=\frac{1}{x^{2}} \frac{d\left(e x^{3}\right)}{d x}-i(i+1) \alpha, \quad z=\frac{1}{x} \frac{d\left(\alpha x^{2}\right)}{d x}-e .
$$

These give

$$
\begin{aligned}
& C_{m}=(m+3) A_{m}-i(i+1) B_{m}, \\
& D_{m}=-A_{m}+(m+2) B_{m},
\end{aligned}
$$

which must hold for all values of $m$ appearing in the assumed series (47). Since the series for $y$ and $z$ contain no terms of degree* $i-1$ or $i-2$, equations (49) require that

$$
\begin{aligned}
& A_{i-2}-i B_{i-2}=0 \\
& A_{i-1}=B_{i-1}=0
\end{aligned}
$$

while for all other values of $m$,

* It will later appear that in the case of fluid equilibrium ( $\mu=0$ and $n=0)$, terms of degree $i-1$ must be introduced unless $k_{1}=0$. This exception has no effect on the $\mathrm{g} \quad . .9$ method of solution. 


$$
\begin{aligned}
& A_{m}=\frac{(m+2) C_{m}+i(i+1) D_{m}}{(m+2)(m+3)-i(i+1)}, \\
& B_{m}=\frac{C_{m}+(m+3) D_{m}}{(m+2)(m+3)-i(i+1)} .
\end{aligned}
$$

Introducing now the notation

$$
k^{\prime}=\rho_{0} / \rho_{m}, \quad b=g \rho_{m} a / \mu,
$$

we proceed to substitute the assumed values of $e, \alpha, y, z$ in (44) and (45).

The expression for $R$ takes the form

in which

$$
\begin{aligned}
L_{i}=\frac{3}{2 i+1} \sum_{m=i}^{\infty}\left(C_{m}-(m-i+2) A_{m}\right)\left(\frac{1}{m-i+2}+\frac{k_{1}}{m-i+3}\right. \\
\left.+\frac{k_{2}}{m-i+4}+\cdots\right)+\frac{2 i-2}{2 i+1} A_{i-2}-\frac{c}{2 k^{\prime}},
\end{aligned}
$$

while for $m=i+1, i+2, \cdots$

$$
R=\rho_{0} \sum_{m=i}^{\infty} L_{m} x^{m}
$$

$$
L_{m}=-\frac{3}{(m-i)(m+i+1)}\left(C_{m-2}+k_{1} C_{m-3}+k_{2} C_{m-4}+\cdots\right.
$$

$$
\begin{aligned}
& \left.+k_{1} A_{m-3}+2 k_{2} A_{m-4}+3 k_{3} A_{m-5}+\cdots\right) \\
& +3\left(\frac{1}{3} A_{m-2}+\frac{1}{4} k_{1} A_{m-3}+\frac{1}{5} k_{2} A_{m-4}+\cdots\right) .
\end{aligned}
$$

Equations (44) become

$$
\begin{aligned}
& \sum\left[\left(\frac{\lambda}{\mu}+2\right) m C_{m}+i(i+1) D_{m}\right] x^{m} \\
& =b{k^{\prime}}^{2}\left(1+k_{1} x+k_{2} x^{2}+\cdots\right)\left[\sum m L_{m} x^{m}-3 \sum C_{m}\left(\frac{1}{3} x^{m+2}\right.\right. \\
& \left.\left.+\frac{1}{4} k_{1} x^{m+3}+\cdots\right)-\frac{n}{k^{\prime}} \sum A_{m} x^{m+2}\right] \text {, } \\
& \sum\left[\left(\frac{\lambda}{\mu}+2\right) C_{m}+(m+1) D_{m}\right] x^{m} \\
& =b{k^{\prime}}^{2}\left(1+k_{1} x+k_{2} x^{2}+\cdots\right)\left[\sum L_{m} x^{m}-\frac{n}{k^{\prime}} \sum B_{m} x^{m+2}\right] \text {; }
\end{aligned}
$$

the summations extending to all values of $m$ occurring in the assumed expansions (47). These equations are to be satisfied identically.

The right-hand members contain no term of degree lower than $i$. The same is true of the left-hand members if (50) and (51) are satisfied. Hence to satisfy (57) identically it is necessary and sufficient to satisfy the following 
equations for $m=i, i+1, i+2, \cdots$ :

$$
\begin{aligned}
& \left(\frac{\lambda}{\mu}+2\right) m C_{m}+i(i+1) D_{m}=b{k^{\prime}}^{2}\left[m L_{m}+k_{1}(m-1) L_{m-1}\right. \\
& +k_{2}(m-2) L_{m-2}+\cdots-\left(C_{m-2}+k_{1}^{\prime} C_{m-3}+k_{2}^{\prime} C_{m-4}+\cdots\right) \\
& \left.\quad-\frac{n}{k^{\prime}}\left(A_{m-2}+k_{1} A_{m-3}+k_{2} A_{m-4}+\cdots\right)\right], \\
& \left(\frac{\lambda}{\mu}+2\right) C_{m}+(m+1) D_{m}=b{k^{\prime 2}}^{\prime 2}\left[L_{m}+k_{1} L_{m-1}+k_{2} L_{m-2}+\cdots\right. \\
& \left.\quad-\frac{n}{k^{\prime}}\left(B_{m-2}+k_{1} B_{m-3}+k_{2} B_{m-4}+\cdots\right)\right],
\end{aligned}
$$

in which $k_{1}^{\prime}, k_{2}^{\prime}, \cdots$ are easily-determined functions of $k_{1}, k_{2}, \cdots$.

For $\dot{m}=i$ the two equations are identical:

$$
\left(\frac{\lambda}{\mu}+2\right) C_{i}+(i+1) D_{i}=b{k^{\prime}}^{2}\left(L_{i}-\frac{n}{i k^{\prime}} A_{i-2}\right)
$$

while for $m=i+1, i+2, \cdots$, they may be solved for $C_{m}$ and $D_{m}$, giving

$$
\begin{gathered}
(m-i)(m+i+1) D_{m}=b{k^{\prime}}^{2}\left[k_{1} L_{m-1}+2 k_{2} L_{m-2}+3 k_{3} L_{m-3}\right. \\
+\cdots+C_{m-2}+k_{1}^{\prime} C_{m-3}+k_{2}^{\prime} C_{m-4}+\cdots+\frac{n}{k^{\prime}}\left(A_{m-2}-m B_{m-2}\right. \\
\left.\left.+k_{1}\left(A_{m-3}-m B_{m-3}\right)+k_{2}\left(A_{m-4}-m B_{m-4}\right)+\cdots\right)\right], \\
\left(\frac{\lambda}{\mu}+2\right) C_{m}=-(m+1) D_{m}+b k^{\prime 2}\left[L_{m}+k_{1} L_{m-1}+k_{2} L_{m-9}\right. \\
\left.\quad+\cdots-\frac{n}{k^{\prime}}\left(B_{m-2}+k_{1} B_{m-3}+k_{2} B_{m-4}+\cdots\right)\right] .
\end{gathered}
$$

These equations serve to determine any pair of coefficients $C_{n}, D_{m}$ (except $\left.C_{i}, D_{i}\right)$ in terms of coefficients of lower order. Remembering the relations (50), (51), (52), (55), and (56), it is seen that every $C_{m}$ and $D_{m}$ may, by successive applications of $(60)$, be ultimately expressed in terms of the three coefficients $C_{i}, D_{i}, A_{i-2}$. When this has been accomplished the only non-vanishing terms in (57) will be those of degree $i$; and these will vanish when (59) is satisfied. Equation (59) in fact becomes one of three linear equations for determining $C_{i}, D_{i}$, and $A_{i-2}$; the other two being obtained from the surface conditions (46). These three equations may be written as follows: 


$$
\begin{aligned}
&\left(\frac{\lambda}{\mu}+2\right) C_{i}+(i+1) D_{i}=b k^{\prime 2}\left[\frac{3}{2 i+1} \sum_{m=i}^{\infty}\left(C_{m}-(m-i+2) A_{m}\right)\right. \\
& \times\left(\frac{1}{m-i+2}+\frac{k_{1}}{m-i+3}+\frac{k_{2}}{m-i+4}+\cdots\right) \\
&\left.+\frac{2 i-2}{2 i+1} A_{i-2}-\frac{c}{2 k^{\prime}}-\frac{n}{i k^{\prime}} A_{i-2}\right], \\
&\text { (61) } \left.\quad \mu \sum_{m=i}^{\infty} C_{m}+2 \mu \sum_{m=i-2}^{\infty}(m+1) A_{m}=0, \quad m B_{m}+A_{m}\right)=0 .
\end{aligned}
$$

The foregoing solution is valid for any constant values of $\lambda$ and $\mu$ which are compatible with the assumption that the strain is "small," i.e., that only the first powers of the displacements are of appreciable magnitude. The statement of the solution however requires modification in the limiting cases of incompressibility $(\lambda=\infty)$ and fluidity $(\mu=0)$.

2. Case of incompressibility. If $\lambda=\infty$ the cubical expansion $\Delta$ must be 0 , hence in applying the general solution to this case we must put $y=0$ while $\lambda y$ remains finite. Putting

$$
\frac{\lambda}{\mu} y=y^{\prime}
$$

equations (44) and (45) become

$$
\begin{gathered}
x \frac{d y^{\prime}}{d x}+i(i+1) z=\frac{g \rho a}{\mu \rho_{m}}\left(x \frac{d R}{d x}-n \rho_{m} e x^{2}\right), \\
y^{\prime}+\frac{d(x z)}{d x}=\frac{g \rho a}{\mu \rho_{m}}\left(R-n \rho_{m} \alpha x^{2}\right), \\
R=-\frac{3}{2 i+1}\left[\frac{1}{x^{i+1}} \int_{0}^{x} \rho \frac{d}{d x}\left(e x^{i+3}\right) d x+x^{i} \int_{x}^{1} \rho \frac{d}{d x}\left(\frac{e}{x^{i-2}}\right) d x\right] \\
+\frac{3 e}{x} \int_{0}^{x} \rho x^{2} d x-\frac{1}{2} c \rho_{m} x^{i}
\end{gathered}
$$

Without restricting the law of density, a linear differential equation for determining $e$ may be obtained by eliminating $y^{\prime}$ between the two equations (63), substituting for $z$ and $\alpha$ their values in terms of $e$ as given by (48) with $y=0$, and substituting for $R$ its value given by (64). By so operating as to eliminate the integrals containing $e$, there results a linear differential equation of the sixth order whose coefficients depend upon $\rho$ and its derivatives. ${ }^{*}$ This equation will not be given; it may be noted, however, that the elimination of the integrals containing $e$ is accomplished by operating upon (64) as indicated

\footnotetext{
* In the case $n=0$ this equation becomes identical with that obtained by Herglotz in the paper cited above. His method of procedure is, however, quite different.
}

Trans. Am. Math. Boc. 8 
in the following equation:

$$
\begin{aligned}
x^{2} \frac{d^{2} R}{d x^{2}}+2 x & \frac{d R}{d x}-i(i+1) R \\
& =3\left(x \frac{d^{2} e}{d x^{2}}-i(i+1) \frac{e}{x}\right) \int_{0}^{x} \rho x^{2} d x+6 \rho x^{2}\left(x \frac{d e}{d x}+e\right) .
\end{aligned}
$$

When the density function is rational and integral, the above method of solution by infinite series applies with slight modification to the case of incompressibility, the equations expressing the solution being in fact much simplified.

Assume

$$
y^{\prime}=\sum_{m=i}^{\infty} K_{m} x^{m}
$$

and equate to 0 every term in the equations which contains $C_{m}$ unless multiplied by $\lambda$, but

$$
\frac{\lambda}{\mu} C_{m}=K_{m}
$$

The main equations in the general solution then take the following forms.

$$
L_{i}=-\frac{3}{2 i+1} \sum_{m=i}^{\infty}(m-1+2) A_{m}\left(\frac{1}{m-i+2}+\frac{k_{1}}{m-i+3}\right.
$$

$$
\left.+\frac{k_{2}}{m-i+4}+\cdots\right)+\frac{2 i-2}{2 i+1} A_{i-2}-\frac{c}{2 k^{\prime}} \text {, }
$$

$$
L_{m}=3\left(\frac{1}{3} A_{m-2}+\frac{1}{4} k_{1} A_{m-3}+\frac{1}{5} k_{2} A_{m-4}+\cdots\right)
$$

$$
-\frac{3}{(m-i)(m+i+1)}\left(k_{1} A_{m-3}+2 k_{2} A_{m-4}+3 k_{3} A_{m-5}+\cdots\right),
$$

$$
m K_{m}+i(i+1) D_{m}=b k^{\prime 2}\left[m L_{m}+k_{1}(m-1) L_{m-1}+k_{2}(m-2) L_{m-2}\right.
$$

$$
\left.+\cdots-\frac{n}{k^{\prime}}\left(A_{m-2}+k_{1} A_{m-3}+k_{2} A_{m-4}+\cdots\right)\right],
$$

(70)

$$
\begin{aligned}
K_{m}+(m+1) D_{m}=b k^{\prime 2} & {\left[L_{m}+k_{1} L_{m-1}+k_{2} L_{m-2}+\cdots\right.} \\
& \left.-\frac{n}{k^{\prime}}\left(B_{m-2}+k_{1} B_{m-3}+k_{2} B_{m-1}+\cdots\right)\right],
\end{aligned}
$$

$$
K_{i}+(i+1) D_{i}=b k^{\prime 2}\left(L_{i}-\frac{n}{i k^{\prime}} A_{i-2}\right),
$$


(72)

$$
\begin{aligned}
& (m-i)(m+i+1) D_{m}=b{k^{\prime}}^{2}\left[k_{1} L_{m-1}+2 k_{2} L_{m-2}+3 k_{3} L_{m-3}\right. \\
& +\cdots+\frac{n}{k^{\prime}}\left(A_{m-2}-m B_{m-2}+k_{1}\left(A_{m-3}-m B_{m-3}\right)+\cdots\right), \\
& K_{m}=-(m+1) D_{m}+b{k^{\prime}}^{2}\left[L_{m}+k_{1} L_{m-1}+k_{2} L_{m-2}+k_{3} L_{m-3}\right. \\
& \left.+\cdots-\frac{n}{k^{\prime}}\left(B_{m-2}+k_{1} B_{m-3}^{\prime}+\cdots\right)\right] \text {. }
\end{aligned}
$$

These correspond respectively to equations (55), (56), (58), (59), and (60).

Proceeding as before, equations (72) serve to determine every $K_{m}$ and $D_{m}$ in terms of $K_{i}, D_{i}$, and $A_{i-2}$. The three equations for determining these (corresponding to (61)) are

(73)

$$
\begin{gathered}
K_{i}+(i+1) D_{i}=b k^{\prime 2}\left[-\frac{3}{2 i+1} \sum_{m=i}^{\infty}(m-i+2)\left(\frac{1}{m-i+2}+\right.\right. \\
\left.\left.\frac{k_{1}}{m-i+3}+\frac{k_{2}}{m-i+4}+\cdots\right) A_{m}+\left(\frac{2 i-2}{2 i+1}-\frac{n}{i k^{\prime}}\right) A_{i-2}-\frac{c}{2 k^{\prime}}\right], \\
\sum_{m=i}^{\infty} K_{m}+2 \sum_{m=i-2}^{\infty}(m+1) A_{m}=0, \quad \mu \sum_{m=i-2}^{\infty}\left(m B_{m}+A_{m}\right)=0 .
\end{gathered}
$$

3. Case of fluidity or zero rigidity. If $\mu=0$, both $\lambda / \mu$ and $b$ become infinite, but their ratio is finite. Hence we multiply equations (57) by $\mu / \lambda$ and introduce the notation

$$
b^{\prime}=\frac{\mu b}{\lambda}=\frac{g \rho_{m} a}{\lambda}
$$

The first members of these equations become

$$
\sum m C_{m} x^{m} \quad \text { and } \quad \sum C_{m} x^{m},
$$

while the second members are unchanged except by the substitution of $b^{\prime}$ for $b$. Equations (58) therefore become

$$
\begin{aligned}
m C_{m}=b^{\prime} k^{\prime 2}\left[m L_{m}+k_{1}(m\right. & -1) L_{m-1}+k_{2}(m-2) L_{m-2}+\cdots \\
& -\left(C_{m-2}+k_{1}^{\prime} C_{m-3}+k_{2}^{\prime} C_{m-4}+\cdots\right) \\
& \left.-\frac{n}{k^{\prime}}\left(A_{m-2}+k_{1} A_{m-3}+k_{2} A_{m-4}+\cdots\right)\right], \\
C_{m}=b^{\prime} k^{\prime 2}\left[L_{m}+k_{1} L_{m-1}\right. & +k_{2} L_{m-2}+\cdots \\
& \left.-\frac{n}{k^{\prime}}\left(B_{m-2}+k_{1} B_{m-3}+k_{2} B_{m-4}+\cdots\right)\right],
\end{aligned}
$$


which must be satisfied for $m=i, i+1, \cdots$. As before, these equations are identical when $m=i$, giving

$$
C_{i}=b^{\prime} k^{\prime 2}\left(L_{i}-\frac{n A_{i-2}}{i k^{\prime}}\right)
$$

corresponding to (59); while for other values of $m$ they must be solved simultaneously. The simplest procedure seems to be to eliminate $C_{m}$ as before, giving an equation similar to the first of (60) but with the first member zero. If the solution is carried out with successive values $m=i+1, i+2, \cdots$, having due regard for equations (50), (51), and (52), every coefficient may be determined in terms of those of lower order, and ultimately in terms of those of lowest order. It is found, however, that when (76) are satisfied for $m=i+1, i+2, \cdots$, only two coefficients remain arbitrary; all coefficients may in fact be determined in terms of $D_{i}$ and $A_{i-2}$ so as to reduce to zero every term in the equations corresponding to (57) except those of degree $i$. In order to cause these to vanish, and also to satisfy the boundary conditions, it is necessary and sufficient to so determine $D_{i}$ and $A_{i-2}$ as to satisfy the equations corresponding to (61). Since $\mu=0$ the third of these is satisfied independently of $D_{i}$ and $A_{i-2}$, while the first and second take the forms

$$
\begin{gathered}
C_{i}=b^{\prime} k^{\prime 2}\left[\frac{3}{2 i+1} \sum_{m=i}^{\infty}\left(C_{m}-(m-i+2) A_{m}\right)\right. \\
\times\left(\frac{1}{m-i+2}+\frac{k_{1}}{m-i+3}+\frac{k_{2}}{m-i+4}+\cdots\right) \\
\left.\quad+\frac{2 i-2}{2 i+1} A_{i-2}-\frac{c}{2 k^{\prime}}-\frac{n}{i k^{\prime}} A_{i-2}\right], \\
\sum_{m=i}^{\infty} C_{m}=0 .
\end{gathered}
$$

Special case of fuid equilibrium. If $n=0$ and $k_{1} \neq 0$, the above solution fails unless terms of degree $i-1$ are introduced in the four series (47). This case is best treated independently, especially as it is possible thereby to show that the above general theory includes as a special case the ordinary theory of the equilibrium of a fluid sphere of variable density.

It is easy to show that, if $n=0$, equations (44) cannot be satisfied with an arbitrary law of density except by making

$$
y=0, \quad R=0 .
$$

Substituting the value of $R$ given by (45) with $y=0$ in the equation

$$
\frac{d}{d x}\left(x^{2} \frac{d R}{d x}\right)-i(i+1) R=0,
$$


and noting equation (65), there results

$$
\frac{i(i+1) e-x^{2} \frac{d^{2} e}{d x^{2}}}{e+x \frac{d e}{d x}}=\frac{2 \rho x^{3}}{\int_{0}^{x} \rho x^{2} d x},
$$

a well-known equation in the theory of equilibrium of a fluid sphere of variable density.

The solution by infinite series when the density function is rational and integral is easily carried out if terms of degree $i-1$ are introduced in the series.* The analysis in fact simplifies greatly from the fact that

$$
C_{m}=0 \quad \text { and } \quad L_{m}=0
$$

for every value of $m$.

IV. SOlution of EQUATIONS FOR CASES OF VARIABLE Elastic MOdULI

1. The differential equations and surface conditions. The differential equations (37) and the surface conditions (39) cover the general case in which $\lambda$ and $\mu$ are any functions of $r$. When $\lambda$ and $\mu$ are constants (37) reduce to (44). Restoring the terms involving derivatives of $\lambda$ and $\mu$, there must be added to the first members of (44) the terms

$$
x y \frac{d \lambda}{d x}+2 x \frac{d(e x)}{d x} \frac{d \mu}{d x}, \quad x\left(e+x \frac{d \alpha}{d x}\right) \frac{d \mu}{d x} .
$$

The surface conditions (46) are unchanged, since they do not involve derivatives of $\lambda$ or $\mu$.

2. Elastic moduli assumed to be rational integral functions of $r$. We now assume that $\lambda$ and $\mu$ are expressible by polynomials in $x$ :

$$
\lambda=\lambda_{0}\left(1+s_{1} x+s_{2} x^{2}+\cdots\right), \quad \mu=\mu_{0}\left(1+t_{1} x+t_{2} x^{2}+\cdots\right),
$$

and proceed to examine the effect of the terms (83) upon the solution by infinite series. The density is still assumed to be given by (40).

The substitution of the assumed series (47) for $y, z, e, \alpha$ reduces the differential equations to two equations similar to (57), but with the addition of terms derived from (83) and (84). If we let

$$
b=g \rho_{m} a / \mu_{0}
$$

(which includes the definition of $b$ given by (53)), the second members of the new equations will be identical with those of (57), while the first members will be the following:

* This is unnecessary if $k_{1}=0$ in the density function. 


$$
\begin{aligned}
& \sum\left\{\left(\frac{\lambda_{0}}{\mu_{0}}+2\right) m C_{m}+i(i+1) D_{m}+\left[\frac{\lambda_{0}}{\mu_{0}} m s_{1}+2(m-1) t_{1}\right] C_{m-1}\right. \\
& \quad+\left[\frac{\lambda_{0}}{\mu_{0}} m s_{2}+2(m-2) t_{2}\right] C_{m-2}+\left[\frac{\lambda_{0}}{\mu_{0}} m s_{3}+2(m-3) t_{3}\right] C_{m-3}+\cdots \\
& \quad+i(i+1)\left(t_{1} D_{m-1}+t_{2} D_{m-2}+\cdots\right) \\
& \left.\quad+2\left[m t_{1} A_{m-1}+2(m-1) t_{2} A_{m-2}+3(m-2) t_{3} A_{m-3}+\cdots\right]\right\} x^{m}, \\
& \sum\left\{\left(\frac{\lambda_{0}}{\mu_{0}}+2\right) C_{m}+(m+1) D_{m}+\left(\frac{\lambda_{0}}{\mu_{0}} s_{1}+2 t_{1}\right) C_{m-1}\right. \\
& \quad+\left(\frac{\lambda_{0}}{\mu_{0}} s_{2}+2 t_{2}\right) C_{m-2}+\left(\frac{\lambda_{0}}{\mu_{0}} s_{3}+2 t_{3}\right) C_{m-3}+\cdots \\
& \quad+m t_{1} D_{m-1}+(m-1) t_{2} D_{m-2}+(m-2) t_{3} D_{m-3}+\cdots \\
& \quad+t_{1}\left[A_{m-1}+(m-1) B_{m-1}\right]+2 t_{2}\left[A_{m-2}+(m-2) B_{m-2}\right] \\
& \left.\quad+3 t_{3}\left[A_{m-3}+(m-3) B_{m-3}\right]+\cdots\right\} x^{m} .
\end{aligned}
$$

These expressions contain no terms of degree lower than $i$, except terms containing $t_{1}$ as a factor. Unless $t_{1}=0$ the series for $y, z, e, \alpha$ must contain terms of degree $i-1$. No terms of degree less than $i+1$ are thereby introduced into the second members of the equations corresponding to (57), while the terms of lowest degree in the first members are

$$
\begin{gathered}
{\left[\left(\frac{\lambda_{0}}{\mu_{0}}+2\right)(i-1) C_{i-1}+i(i+1) D_{i-1}+2(i-1) t_{1} A_{i-2}\right] x^{i-1},} \\
{\left[\left(\frac{\lambda_{0}}{\mu_{0}}+2\right) C_{i-1}+i D_{i-1}+t_{1}\left(A_{i-2}+(i-2) B_{i-2}\right)\right] x^{i-1}}
\end{gathered}
$$

which must be made to vanish by determining $C_{i-1}$ and $D_{i-1}$ in terms of $A_{i-2}$, remembering (50).

In the subsequent analysis it will be assumed that $t_{1}=s_{1}=0,-$ a reasonable assumption in any geodynamical application, since it is natural to suppose that $d \lambda / d x$ and $d \mu / d x$ vanish at the center.

Using (86) instead of the first members of (57), and assuming the new equations to be satisfied identically, we obtain a pair of equations whose second members are identical with those of (58) and whose first members are the following: 
(88)

$$
\begin{aligned}
\left(\frac{\lambda_{0}}{\mu_{0}}+2\right) m C_{m}+i(i+1) D_{m}+\left[\frac{\lambda_{0}}{\mu_{0}} m s_{2}+2(m-2) t_{2}\right] C_{m-2} \\
\quad+\frac{\lambda_{0}}{\mu_{0}}\left[m s_{3}+2(m-3) t_{3}\right] C_{m-3}+\cdots \\
\quad+i(i+1)\left(t_{2} D_{m-2}+t_{3} D_{m-3}+\cdots\right) \\
\quad+2\left[2(m-1) t_{2} A_{m-2}+3(m-2) t_{3} A_{m-3}+\cdots\right], \\
\quad\left(\frac{\lambda_{0}}{\mu_{0}}+2\right) C_{m}+(m+1) D_{m}+\left(\frac{\lambda_{0}}{\mu_{0}} s_{2}+2 t_{2}\right) C_{m-2} \\
\quad+\left(\frac{\lambda_{0}}{\mu_{0}} s_{3}+2 t_{3}\right) C_{m-3}+\cdots+(m-1) t_{2} D_{m-2} \\
\quad+(m-2) t_{3} D_{m-3}+\cdots+2 t_{2}\left[A_{m-2}+(m-2) B_{m-2}\right] \\
\quad+3 t_{3}\left[A_{m-3}+(m-3) B_{m-3}\right]+\cdots .
\end{aligned}
$$

For $m=i$ the two equations are identical, giving

(89) $\left(\frac{\lambda_{0}}{\mu_{0}}+2\right) C_{i}+(i+1) D_{i}+\frac{4(i-1)}{i} t_{2} A_{i-2}=b k^{\prime 2}\left(L_{i}-\frac{n}{i k^{\prime}} A_{i-2}\right)$.

For $m>i$ they may be solved for $C_{m}$ and $D_{m}$, giving these in terms of coefficients of lower order, and thus ultimately in terms of $C_{i}, D_{i}, A_{i-2}$. If the resulting series are convergent, the solution may be completed as in the case of uniform elasticity already treated; i.e., by substituting the values of $C_{m}$ and $D_{m}$ (expressed as linear functions of $C_{i}, D_{i}, A_{i-2}$ ) in (89) and in the boundary equations (46), thus obtaining three linear equations for determining $C_{i}, D_{i}, A_{i-2}$. These equations, corresponding to (61), are the following ( $\lambda_{1}$ and $\mu_{1}$ being surface values of $\lambda$ and $\mu$ ):

$$
\begin{aligned}
& \left(\frac{\lambda_{0}}{\mu_{0}}+2\right) C_{i}+(i+1) D_{i}+\frac{4(i-1)}{i} t_{2} A_{i-2} \\
& \quad=b{k^{\prime}}^{2}\left\{\frac { 3 } { 2 i + 1 } \sum _ { m = i } ^ { \infty } [ C _ { m } - ( m - i + 2 ) A _ { m } ] \left(\frac{1}{m-i+2}\right.\right. \\
& \left.\left.\quad+\frac{k_{1}}{m-i+3}+\cdots\right)+\frac{2 i-2}{2 i+1} A_{i-2}-\frac{c}{2 k^{\prime}}-\frac{n}{i k^{\prime}} A_{i-2}\right\}, \\
& \quad \frac{\lambda_{1}}{\mu_{1}} \sum_{\text {m }}^{\infty} C_{m}+2 \sum_{m=i-2}^{\infty}(m+1) A_{m}=0, \quad \mu_{1} \sum_{m=i-2}^{\infty}\left(m B_{m}+A_{m}\right)=0 .
\end{aligned}
$$

3. Convergency of series. When the above process of solving for $C_{m}$ and $D_{m}$ is carried out, it is probable that the resulting series do not converge unless important restrictions are imposed upon the constants $s_{m}, t_{m}$. The values 
of $C_{m}$ and $D_{m}$, so far as these depend upon terms occurring in (88), are as follows:

$$
\begin{aligned}
& (m-i)(m+i+1) D_{m} \\
& =-t_{2}\left[4 C_{m-2}+\left(m^{2}-m-i^{2}-i\right) D_{m-2}-2(m-2) A_{m-2}\right. \\
& \left.+2 m(m-2) B_{m-2}\right] \\
& -t_{3}\left[6 C_{m-3}+\left(m^{2}-2 m-i^{2}-i\right) D_{m-3}-3(m-4) A_{m-3}\right. \\
& \left.+3 m(m-3) B_{m-3}\right] \\
& -t_{4}\left[8 C_{m-4}+\left(m^{2}-3 m-i^{2}-i\right) D_{m-4}-4(m-6) A_{m-4}\right. \\
& \left.+4 m(m-4) B_{m-4}\right] \\
& (m-i)(m+i+1)\left(\frac{\lambda_{0}}{\mu_{0}}+2\right) C_{m} \\
& =-(m-i)(m+i+1) \frac{\lambda_{0}}{\mu_{0}}\left(s_{2} C_{m-2}+s_{3} C_{m-3}+s_{4} C_{m-4}+\cdots\right) \\
& -t_{2}\left[2\{(m+1)(m-2)-i(i+1)\} C_{m-2}+2 i(i+1) D_{m-2}\right. \\
& +(4(m-1)(m+1)-2 i(i+1)) A_{m-2} \\
& \left.-2 i(i+1)(m-2) B_{m-2}\right] \\
& -t_{3}\left[2\{(m+1)(m-3)-i(i+1)\} C_{m-3}+3 i(i+1) D_{m-3}\right. \\
& +(6(m-2)(m+1)-3 i(i+1)) A_{m-3} \\
& \left.-3 i(i+1)(m-3) B_{m-3}\right] \\
& -t_{4}\left[2\{(m+1)(m-4)-i(i+1)\} C_{m-4}+4 i(i+1) D_{m-4}\right. \\
& +(8(m-3)(m+1)-4 i(i+1)) A_{m-4} \\
& \left.-4 i(i+1)(m-4) B_{m-4}\right]
\end{aligned}
$$

So far as convergence is concerned, the governing terms are those of highest degree in $m$, after every $A_{n}$ and $B_{n}$ has been replaced by its value in terms of $C_{n}$ and $D_{n}$ by equations (52). After division by $(m-i)(m+i+1)$ these governing terms (of degree 0 in $m$ ) are as follows:

$$
\begin{gathered}
D_{m}=-t_{2} D_{m-2}-t_{3} D_{m-3}-t_{4} D_{m-4}-\cdots, \\
C_{m}=-\frac{\lambda_{0} s_{2}+2 \mu_{0} t_{2}}{\lambda_{0}+2 \mu_{0}} C_{m-2}-\frac{\lambda_{0} s_{3}+2 \mu_{0} t_{3}}{\lambda_{0}+2 \mu_{0}} C_{m-3}-\cdots
\end{gathered}
$$


Inspection of these terms shows that it is possible to insure the convergence of the series $C_{m}$ and $D_{m}$ by making all the governing terms zero except one in each equation and making the coefficients in the non-vanishing terms less than unity in absolute value; for in such case each series will ultimately approach coincidence with a convergent geometric series. The solution is thus practicable if $\lambda$ and $\mu$ are expressed by binomial functions

$$
\begin{aligned}
& \lambda=\lambda_{0}\left(1+s_{n} x^{n}\right), \\
& \mu=\mu_{0}\left(1+t_{n} x^{n}\right),
\end{aligned}
$$

in which $n$ is a positive integer and $s_{n}, t_{n}$ are less than unity in absolute value.

Even with this restriction the solution is of very considerable interest, since it supplies a very important generalization in the case of the geodynamical problem of the elastic yielding of the earth.

\section{Particular solutions}

1. Numerical applications. The general solution above given has been applied in the computation of a considerable number of numerical results. The data used in these computations have been chosen in approximate conformity with the known dimensions and properties of the earth, and the results fall into several series designed to show separately the effects of different assumptions regarding density, compressibility, and rigidity. Since the labor increases considerably with each added term in the density formula, the computations have been restricted to the case in which the formula is a binomial. Fortunately this simple formula is capable of expressing a fair approximation to the facts for the earth so far as known. It has already been pointed out that the above solution for variable elasticity is restricted to the case in which $\lambda$ and $\mu$ are expressed by binomials.

While omitting numerical details, it seems desirable to show the form to which the main formulas have been reduced for the purpose of computation. These will be given in a form sufficiently inclusive to cover all the special cases for which computations have been made.

2. Formulas for density and elastic moduli. The simplified formulas assumed for $\rho, \lambda, \mu$ are as follows:

$$
\begin{gathered}
\rho=\rho_{0}\left(1-k x^{2}\right), \\
\lambda=\lambda_{0}\left(1-h x^{2}\right), \quad \mu=\mu_{0}\left(1-h x^{2}\right),
\end{gathered}
$$

in which $k$ and $h$ are positive and less than 1. It will be observed that (95) fall under (93), and also that they make $\lambda / \mu$ constant.

The surface values are

$$
\rho_{1}=\rho_{0}(1-k),
$$




$$
\lambda_{1}=\lambda_{0}(1-h), \quad \mu_{1}=\mu_{0}(1-h),
$$

while the mean density is

so that

$$
\rho_{m}=\rho_{0}\left(1-\frac{3}{5} k\right)
$$

$$
k^{\prime}=\frac{\rho_{0}}{\rho_{m}}=\frac{5}{5-3 k} .
$$

The numerical values to be assigned to the constants $k, h, \rho_{0}, \lambda_{0}, \mu_{0}$ in order to represent as nearly as possible the facts for the earth will be considered later.

3. Disturbing potential of second degree. The particular solutions which will be considered all fall under the case $i=2$, which covers the actual case of tidal and centrifugal forces. The lunar or solar tidal potential is in fact expressed by (29) with

$$
S_{i}=S_{2}=\cos ^{2} \theta^{\prime}-\frac{1}{3},
$$

in which $\theta^{\prime}$ is the zenith distance of the disturbing body. Referring to (42) and (21) it is seen that, with this value of $S_{2}, e$ denotes the ellipticity of a surface which in the unstrained body would be spherical and of radius $r$, while $\alpha$ denotes the angular displacement of a radius vector drawn to a particle for which $\theta^{\prime}=45^{\circ}$.

In the static problem we may take $\theta^{\prime}=\theta$.

4. Differential equations and boundary conditions. We now return to equations (37), (38), and (39), and note the form taken by their solution in the case represented by (96), (97), and (100). After the introduction of $x$ as independent variable the differential equations are

$$
\begin{gathered}
(\lambda+2 \mu) x \frac{d y}{d x}+6 \mu z+x y \frac{d \lambda}{d x}+2 x \frac{d(e x)}{d x} \frac{d \mu}{d x} \\
=\frac{g \rho a}{\rho_{m}}\left(x \frac{d R}{d x}-\frac{3 y}{x} \int_{0}^{x} \rho x^{2} d x-n \rho_{m} e x^{2}\right) \\
(\lambda+2 \mu) y+\mu \frac{d(x z)}{d x}+x\left(e+x \frac{d \alpha}{d x}\right) \frac{d \mu}{d x}=\frac{g \rho a}{\rho_{m}}\left(R-n \rho_{m} \alpha x^{2}\right),
\end{gathered}
$$

in which

$$
R=\frac{3}{5}\left[\frac{1}{x^{3}} \int_{0}^{x} \rho\left(y x^{4}-\frac{d\left(e x^{5}\right)}{d x}\right)+x^{2} \int_{x}^{1} \rho\left(\frac{y}{x}-\frac{d e}{d x}\right) d x\right]
$$

$$
+\frac{3 e}{x} \int_{0}^{x} \rho x^{2} d x-\frac{c \rho_{m} x^{2}}{2}
$$

while the boundary conditions are

$$
\lambda y+2 \mu \frac{d(e x)}{d x}=0, \quad \mu\left(e+x \frac{d \alpha}{d x}\right)=0, \quad(\text { when } x=1)
$$


These are obtained from (44), (45), and (46) by putting $i=2$ and adding the terms (83) to the first members of (44).

Equations (23), defining $y$ and $z$, now become

$$
y=\frac{1}{x^{2}} \frac{d\left(e x^{3}\right)}{d x}-6 \alpha, \quad z=\frac{1}{x} \frac{d\left(\alpha x^{2}\right)}{d x}-e .
$$

5. Solution of equations. In the present case only even powers of $x$ are required in the assumed series (47), which become

$$
\begin{array}{ll}
y=C_{2} x^{2}+C_{4} x^{4}+\cdots & z=D_{2} x^{2}+D_{4} x^{4}+\cdots \\
e=A_{0}+A_{2} x^{2}+A_{4} x^{4}+\cdots & \alpha=B_{0}+B_{2} x^{2}+B_{4} x^{4}+\cdots
\end{array}
$$

Because of (104) the coefficients in (105) are related as follows:

$$
C_{m}=(m+3) A_{m}-6 B_{m}, \quad D_{m}=-A_{m}+(m+2) B_{m} .
$$

This requires that

$$
B_{0}=\frac{1}{2} A_{0},
$$

while for $m=2,4, \cdots, A_{m}$ and $B_{m}$ may be expressed in terms of $C_{m}$ and $D_{m}$ by the formulas

$$
A_{m}=\frac{(m+2) C_{m}+6 D_{m}}{m(m+5)}, \quad B_{m}=\frac{C_{m}+(m+3) D_{m}}{m(m+5)},
$$

which are equations (52) with $i=2$.

The solution now proceeds as outlined in equations (54)-(61), but taking account of the terms which must be added when $\lambda$ and $\mu$ are variable. These terms are obtained from the results given in Part IV, noting that all the constants $s_{m}, t_{m}$ in (84) vanish except $s_{2}, t_{2}$, each of which is now replaced by $-h$.

The value of $R$ becomes

$$
R=\rho_{0}\left(L_{2} x^{2}+L_{4} x^{4}+\cdots\right),
$$

in which

$$
L_{2}=\frac{3}{5} \sum_{m=2}^{\infty}\left(C_{m}-m A_{m}\right)\left(\frac{1}{m}-\frac{k}{m+2}\right)+\frac{2}{5} A_{0}-\frac{c}{2 k^{\prime}},
$$

while for $m=4,6, \cdots$

$$
L_{m}=-\frac{3}{(m-2)(m+3)}\left(C_{m-2}-k C_{m-4}-2 k A_{m-4}\right)+A_{m-2}-\frac{3}{5} k A_{m-4} .
$$

These correspond to equations (54), (55), and (56).

The substitution of (105) in (101) gives the equations corresponding to (57); the second members are in fact identical with those of (57), while the first members are given by (86), proper substitutions being made for $k_{1}, k_{2}$, 
$\cdots, s_{1}, s_{2}, \cdots, t_{1}, t_{2}, \cdots$, and $b$ being defined by (85). These equations are

$$
\begin{aligned}
& \sum\left[\left(\frac{\lambda_{0}}{\mu_{0}}+2\right) m C_{m}+6 D_{m}-\left(m \frac{\lambda_{0}}{\mu_{0}}+2(m-2)\right) h C_{m-2}\right. \\
& \left.-6 h D_{m-2}-4(m-1) h A_{m-2}\right] x^{m} \\
& =b{k^{\prime}}^{2}\left(1-k x^{2}\right) \sum\left[m L_{m} x^{m}-3 C_{m}\left(\frac{1}{3} x^{m+2}-\frac{1}{5} k x^{m+4}\right)\right. \\
& \left.-\frac{n}{k^{\prime}} A_{m} x^{m+2}\right] \text {, } \\
& \sum\left[\left(\frac{\lambda_{0}}{\mu_{0}}+2\right) C_{m}+(m+1) D_{m}-\left(\frac{\lambda_{0}}{\mu_{0}}+2\right) h C_{m-2}\right. \\
& \left.-(m-1) h D_{m-2}-2 h\left(A_{m-2}+(m-2) B_{m-2}\right)\right] x^{m} \\
& =b{k^{\prime}}^{2}\left(1-k x^{2}\right) \sum\left[L_{m} x^{m}-\frac{n}{k^{\prime}} B_{m} x^{m+2}\right] \text {, }
\end{aligned}
$$

the summations extending to all values of $m$ which are consistent with (105). Both equations thus take the form of series of even powers of $x$, beginning with $x^{2}$. To satisfy them identically it is necessary to satisfy the following equations for $m=2,4,6, \cdots$ :

$$
\begin{aligned}
& \left(\frac{\lambda_{0}}{\mu_{0}}+2\right) m C_{m}+6 D_{m}-h\left[\left(\frac{\lambda_{0}}{\mu_{0}} m+2(m-2)\right) C_{m-2}\right. \\
& \left.+6 D_{m-2}+4(m-1) A_{m-2}\right] \\
& =b{k^{\prime}}^{2}\left[m L_{m}-k(m-2) L_{m-2}-C_{m-2}+\frac{8}{5} k C_{m-4}-\frac{3}{5} k^{2} C_{m-6}\right. \\
& \left.-\frac{n}{k^{\prime}}\left(A_{m-2}-k A_{m-4}\right)\right] \text {, } \\
& \left(\frac{\lambda_{0}}{\mu_{0}}+2\right) C_{m}+(m+1) D_{m}-h\left[\left(\frac{\lambda_{0}}{\mu_{0}}+2\right) C_{m-2}+(m-1) D_{m-2}\right. \\
& \left.+2 A_{m-2}+2(m-2) B_{m-2}\right] \\
& =b k^{\prime 2}\left[L_{m}-k L_{m-2}-\frac{n}{k^{\prime}}\left(B_{m-2}-k B_{m-4}\right)\right] \text {. }
\end{aligned}
$$

For $m=2$ the two equations are identical, giving

$$
\left(\frac{\lambda_{0}}{\mu_{0}}+2\right) C_{2}+3 D_{2}-2 h A_{0}=b{k^{\prime}}^{2}\left(L_{2}-\frac{n}{2 k^{\prime}} A_{0}\right) ;
$$

while for $m=4,6, \cdots$, they may be solved for $C_{m}$ and $D_{m}$, giving 


$$
\begin{array}{r}
D_{m}=\frac{b{k^{\prime 2}}^{2}}{(m-2)(m+3)}\left[-2 k L_{m-2}+C_{m-2}-\frac{8}{5} k C_{m-4}+\frac{3}{5} k^{2} C_{m-6}\right. \\
\left.+\frac{n}{k^{\prime}}\left(A_{m-2}-m B_{m-2}-k\left(A_{m-4}-m B_{m-4}\right)\right)\right] \\
\quad+h\left(D_{m-2}+\frac{4 C_{m-2}-4 D_{m-2}}{(m-2)(m+3)}\right) \\
\left(\frac{\lambda_{0}}{\mu_{0}}+2\right) C_{m}=-(m+1) D_{m}+b{k^{\prime}}^{2}\left[L_{m}-k L_{m-2}\right. \\
\left.\quad-\frac{n}{k^{\prime}}\left(B_{m-2}-k B_{m-4}\right)\right]+h\left[\left(\frac{\lambda_{0}}{\mu_{0}}+2\right) C_{m-2}\right. \\
\left.\quad+\frac{4(m-1) C_{m-2}+\left(m^{3}+2 m^{2}-9 m+14\right) D_{m-2}}{(m-2)(m+3)}\right] .
\end{array}
$$

It should be noted that, in the terms containing $h, A_{m-2}$, and $B_{m-2}$ have been replaced by $C_{m-2}$ and $D_{m-2}$ by means of (108). In applying these equations, $L_{m}$ and $L_{m-2}$ are to be replaced by their values as given by (111), except in the case of $L_{2}$ which is to be eliminated by means of (114). In this way every $C_{m}$ and $D_{m}$ may be computed in terms of coefficients of lower order, and ultimately in terms of $C_{2}, D_{2}, A_{0}$.

For purposes of computation the formulas for $C_{m}$ and $D_{m}$ may conveniently be expressed explicitly in terms of like coefficients of the three preceding orders. This is possible when $m>6$; when $m=4$ and 6 special formulas are required. Convenient working formulas may be written as follows:

(116)

$$
\begin{aligned}
D_{4}=\frac{b k^{\prime 2}}{14}\left(C_{2}-\frac{n}{k^{\prime}} D_{2}\right)-\frac{k}{7}\left[\left(\frac{\lambda_{0}}{\mu_{0}}+2\right) C_{2}+3 D_{2}-2 h A_{0}\right] \\
+\frac{h}{7}\left(2 C_{2}+5 D_{2}\right),
\end{aligned}
$$

$$
\left(\frac{\lambda_{0}}{\mu_{0}}+2\right) C_{4}=\frac{b k^{\prime 2}}{14}\left(-4 C_{2}+6 D_{2}-\frac{12 k}{5} A_{0}-\frac{n}{k^{\prime}} C_{2}\right)
$$

$-\frac{2 k}{7}\left[\left(\frac{\lambda_{0}}{\mu_{0}}+2\right) C_{2}+3 D_{2}-2 h A_{0}\right]+h\left[\left(\frac{\lambda_{0}}{\mu_{0}}+2\right) C_{2}-\frac{4}{7}\left(C_{2}-3 D_{2}\right)\right]$.

$$
D_{6}=\frac{b k^{\prime 2}}{36}\left[C_{4}-\frac{k}{35}\left(61 C_{2}+30 D_{2}\right)+\frac{12 k^{2}}{35} A_{0}\right.
$$

$$
\left.-\frac{n}{k^{\prime}}\left(D_{4}-\frac{k}{7}\left(C_{2}+12 D_{2}\right)\right)\right]+\frac{h}{9}\left(C_{4}+8 D_{4}\right),
$$

$$
\left(\frac{\lambda_{0}}{\mu_{0}}+2\right) C_{6}=\frac{b k^{\prime 2}}{36}\left[-4 C_{4}+6 D_{4}+\frac{k}{35}\left(286 C_{2}-564 D_{2}\right)\right.
$$

$\left.\left.+\frac{132 k^{2}}{35} A_{0}-\frac{n}{k^{\prime}}\left(C_{4}-\frac{k}{7}\left(11 C_{2}+6 D_{2}\right)\right)\right]+h\left[\left(\frac{\lambda_{0}}{\mu_{0}}+2\right) C_{4}-\frac{2}{9}\left(C_{4}-3 D_{4}\right)\right]\right)$. 
For $m=8,10, \cdots$,

$$
\begin{aligned}
D_{m}= & b k^{\prime 2}\left[\frac{C_{m-2}}{(m-2)(m+3)}-k \frac{2\left(4 m^{2}-7 m-41\right) C_{m-4}+60 D_{m-4}}{5(m-4)(m-2)(m+1)(m+3)}\right. \\
& +k^{2} \frac{3\left(m^{4}-8 m^{3}-m^{2}+76 m+28\right) C_{m-6}+36\left(m^{2}-3 m-4\right) D_{m-6}}{5(m-6)(m-4)(m-2)(m-1)(m+1)(m+3)} \\
& \left.-\frac{n}{k^{\prime}}\left(\frac{D_{m-2}}{(m-2)(m+3)}-k \frac{2 C_{m-4}+\left(m^{2}-m-6\right) D_{m-4}}{(m-4)(m-2)(m+1)(m+3)}\right)\right] \\
& +h\left(D_{m-2}+\frac{4 C_{m-2}-4 D_{m-2}}{(m-2)(m+3)}\right), \\
\left(\frac{\lambda_{0}}{\mu_{0}}+\right. & 2) C_{m}=b k^{\prime 2}\left[\frac{-4 C_{m-2}+6 D_{m-2}}{(m-2)(m+3)}\right. \\
& +k \frac{4\left(8 m^{2}-8 m-97\right) C_{m-4}-12\left(4 m^{2}-m-44\right) D_{m-4}}{5(m-4)(m-2)(m+1)(m+3)} \\
& -k^{2} \frac{12\left(m^{4}-6 m^{3}-14 m^{2}+69 m+46\right) C_{m-6}}{5(m-6)(m-4)(m-2)(m-1)(m+1)(m+3)} \\
& \left.-\frac{n}{k^{\prime}}\left(\frac{C_{m-2}}{(m-2)(m+3)}-k \frac{\left(m^{2}-m-8\right) C_{m-4}+12 D_{m-4}}{(m-4)(m-2)(m+1)(m+3)}\right)\right] \\
& +h\left[\left(\frac{\lambda_{0}}{\mu_{0}}+2\right) C_{m-2}-\frac{8 C_{m-2}-24 D_{m-2}}{(m-2)(m+3)}\right] .
\end{aligned}
$$

It is seen that, in (118), the governing terms as regards convergency are those of degree 0 in $m$. Thus the formulas ultimately approach the forms

$$
D_{m}=h D_{m-2}, \quad C_{m}=h C_{m-2},
$$

which indicate convergency if $h<1$; this of course includes the case of uniform elasticity $(h=0)$.

It remains to satisfy (114) and the boundary conditions (103), $L_{2}$ being replaced by its value (110). These reduce to the following three equations, which, after substitution of the values of the summations, become linear equations in $C_{2}, D_{2}, A_{0}$ :

$$
\begin{aligned}
\left(\frac{\lambda_{0}}{\mu_{0}}+2\right) C_{2}+3 D_{2}-2 h A_{0} \\
=b k^{\prime 2}\left[\frac{9}{25}\left(\sum_{2}^{\infty} \frac{C_{m}-2 D_{m}}{m}-\sum_{2}^{\infty} \frac{C_{m}-2 D_{m}}{m}\right)\right. \\
\left.\quad-\frac{3}{5} k\left(\sum_{2}^{\infty} \frac{C_{m}-2 D_{m}}{m+2}-\sum_{2}^{\infty} \frac{C_{m}-2 D_{m}}{m+5}\right)+\left(\frac{2}{5}-\frac{n}{2 k^{\prime}}\right) A_{0}-\frac{c}{2 k^{\prime}}\right],
\end{aligned}
$$




$$
\begin{gathered}
\left(\frac{\lambda_{0}}{\mu_{0}}+2\right) \sum_{2}^{\infty} C_{m}+\frac{4}{5} \sum_{2}^{\infty} \frac{C_{m}+3 D_{m}}{m}-\frac{24}{5} \sum_{2}^{\infty} \frac{C_{m}-2 D_{m}}{m+5}+2 A_{0}=0, \\
\sum_{2}^{\infty} D_{m}+\frac{2}{5} \sum_{2}^{\infty} \frac{C_{m}+3 D_{m}}{m}+\frac{8}{5} \sum_{2}^{\infty} \frac{C_{m}-2 D_{m}}{m+5}+A_{0}=0 .
\end{gathered}
$$

The cases for which numerical results have been computed are all covered by the foregoing equations; the specific formulas for each case being easily written by assigning particular values to $k, h$, and $n$.

6. Case of uniform density and elasticity. For the purpose of comparison with previously known solutions, it is of interest to show the form assumed by the main working formulas in the case $h=0, k=0$, both for unrestricted compressibility and for $\lambda=\infty$.

(A) Compressibility unrestricted. Putting $h=0, k=0, k^{\prime}=1$, equations (118) become

$$
\begin{aligned}
D_{m} & =\frac{b}{(m-2)(m+3)}\left(C_{m-2}-n D_{m-2}\right), \\
\left(\frac{\lambda}{\mu}+2\right) C_{m} & =\frac{b}{(m-2)(m+3)}\left(-(4+n) C_{m-2}+6 D_{m-2}\right),
\end{aligned}
$$

and hold for $m=4,6,8, \cdots$. Successive applications of these equations determine every $C_{m}$ and $D_{m}$ in terms of $C_{2}$ and $D_{2}$. These values are to be substituted in (120), of which the second and third are unchanged while the first simplifies as follows:

$$
\begin{aligned}
\left(\frac{\lambda}{\mu}+2\right) C_{2}+3 D_{2}=b\left[\frac { 9 } { 2 5 } \left(\sum_{2}^{\infty} \frac{C_{m}-2 D_{m}}{m}-\right.\right. & \left.\sum_{2}^{\infty} \frac{C_{m}-2 D_{m}}{m+5}\right) \\
& \left.+\left(\frac{2}{5}-\frac{n}{2}\right) A_{0}-\frac{c}{2}\right] .
\end{aligned}
$$

(B) Incompressibility. When $\lambda=\infty$ we proceed as indicated in the general solution, equations (63)-(69). Equations (121) then become

$$
D_{m}=-\frac{b n D_{m-2}}{(m-2)(m+3)}, \quad K_{m}=\frac{6 b D_{m-2}}{(m-2)(m+3)},
$$

while (120) reduce to the following:

$$
\begin{aligned}
K_{2}+3 D_{2}= & b\left[\frac{18}{25}\left(\sum_{2}^{\infty} \frac{D_{m}}{m+5}-\sum_{2}^{\infty} \frac{D_{m}}{m}\right)+\left(\frac{2}{5}-\frac{n}{2}\right) A_{0}-\frac{c}{2}\right], \\
& \sum_{2}^{\infty} K_{m}+\frac{12}{5} \sum_{2}^{\infty} \frac{D_{m}}{m}+\frac{48}{5} \sum_{2}^{\infty} \frac{D_{m}}{m+5}+2 A_{0}=0 \\
& \sum_{2}^{\infty} D_{m}+\frac{6}{5} \sum_{2}^{\infty} \frac{D_{m}}{m}-\frac{16}{5} \sum_{2}^{\infty} \frac{D_{m}}{m+5}+A_{0}=0
\end{aligned}
$$


Static problem. When $n=0$ equations (123) and (124) result in the wellknown solution for the static strain of a homogeneous incompressible sphere. Thus equations (123) show that; if $n=0$, every $D_{m}$ vanishes except $D_{2}$, that every $K_{m}$ vanishes except $K_{2}$ and $K_{4}$, and that

$$
K_{4}=\frac{3}{7} b D_{2} \text {. }
$$

Equations (124) are thus reduced to the following:

$$
\begin{aligned}
K_{2}+\left(3+\frac{9}{35} b\right) D_{2}-\frac{2}{5} b A_{0}+\frac{1}{2} b c & =0, \\
K_{2}+\left(\frac{18}{7}+\frac{3}{7} b\right) D_{2}+2 A_{0} & =0, \\
\frac{8}{7} D_{2}+A_{0} & =0 ;
\end{aligned}
$$

from which

$$
\frac{A_{0}}{c}=\frac{8 b}{38+4 b}, \quad \frac{D_{2}}{c}=-\frac{7 b}{38+4 b}, \quad \frac{K_{2}}{c}=\frac{(2+3 b) b}{38+4 b} .
$$

Also, from (107) and (108),

$$
\begin{aligned}
& \frac{A_{2}}{c}=\frac{3}{7} \frac{D_{2}}{c}=-\frac{3 b}{38+4 b}, \\
& \frac{B_{2}}{c}=\frac{5}{14} \frac{D_{2}}{c}=-\frac{5 b}{2(38+4 b)}, \\
& \frac{B_{0}}{c}=\frac{A_{0}}{2 c}=\frac{4 b}{38+4 b},
\end{aligned}
$$

so that the final solution is

$$
\frac{e}{c}=\frac{8-3 x^{2}}{38 \frac{\mu}{g \rho a}+4}, \quad \frac{\alpha}{c}=\frac{8-5 x^{2}}{2\left(38 \frac{\mu}{g \rho a}+4\right)},
$$

the known solution for the static problem.

Oscillation of an incompressible fluid sphere. It is of interest to note the simple form assumed by the solution in the case $\rho=$ constant, $\lambda=\infty$, $\mu=0, n \neq 0$. This is best treated by direct use of equations (44), (45), and (46), noting that $y=0$ but $\lambda y \neq 0$, and that the second of (46) is satisfied by the condition $\mu=0$. The simplified equations are the following:

$$
\begin{gathered}
x \frac{d(\lambda y)}{d x}=g a\left(x \frac{d R}{d x}-n \rho e x^{2}\right), \\
\lambda y=g a\left(R-n \rho \alpha x^{2}\right),
\end{gathered}
$$




$$
\begin{array}{ccc}
R & =\rho\left(e-\frac{3}{5} e_{1}-\frac{1}{2} c\right) x^{2}, \\
\lambda y & =0 \quad \text { when } \quad x=1 .
\end{array}
$$

Equations (130) give

$$
x \frac{d\left(\alpha x^{2}\right)}{d x}-e x^{2}=0 .
$$

But since $y=0$ the first of (48) gives

$$
6 \alpha x^{2}=\frac{d\left(e x^{3}\right)}{d x} .
$$

Rejecting negative powers of $x$ as irrelevant to the physical problem, equations (133) and (134) give

$$
e=2 \alpha=\text { constant }=e_{1} .
$$

Hence

$$
\begin{gathered}
R=\rho\left(\frac{2}{5} e_{1}-\frac{1}{2} c\right) x^{2}, \\
\lambda y=g \rho a\left(\left(\frac{2}{5}-\frac{n}{2}\right) e_{1}-\frac{1}{2} c\right) x^{2} .
\end{gathered}
$$

Finally, from the surface condition (132),

$$
\frac{e}{c}=\frac{e_{1}}{c}=\frac{5}{4-5 n} \text {. }
$$

Numerical applications of this equation will be given later.

7. Quantities measuring the strain. Two quantities are of especial importance because their values enter into the observational data from which the actual strain of the earth is inferred. One of these is the surface ellipticity $e_{1}$, while the other depends upon the change in the principal moments of inertia caused by the strain.

Surface ellipticity. This may be computed by the formula

$$
e_{1}=\sum_{m=0}^{\infty} A_{m}=A_{0}+\frac{2}{5} \sum_{m=2}^{\infty} \frac{C_{m}+3 D_{m}}{m}+\frac{3}{5} \sum_{m=2}^{\infty} \frac{C_{m}-2 D_{m}}{m+5},
$$

obtained by the use of (108).

Change of principal moments of inertia. Let $C, A, A$ be the principal moments of inertia of the strained body (two of them being equal since $S_{2}$ has an axis of symmetry). Then it may be shown that

$$
A-C=-\frac{8 \pi a^{5}}{15} \int_{0}^{1} \rho\left(y x^{4}-\frac{d\left(e x^{5}\right)}{d x}\right) d x,
$$

which, by the use of (104), may be written

$$
A-C=\frac{\mathrm{i} 6 \pi a^{5}}{15} \int_{0}^{1} \rho(e+3 \alpha) x^{4} d x .
$$

Trans. Am. Math. Soc. 3 
Let $I$ denote the diametral moment of inertia of the unstrained body, and let

$$
f=\frac{A-C}{I} .
$$

Then

$$
I=\frac{8 \pi}{3} \int_{0}^{a} \rho r^{4} d r=\frac{8 \pi a^{5}}{3} \int_{0}^{1} \rho x^{4} d x
$$

so that

$$
f=\frac{2 \int_{0}^{1} \rho(e+3 \alpha) x^{4} d x}{5 \int_{0}^{1} \rho x^{4} d x} .
$$

Using the value of $\rho$ given by (94), and substituting the values of $e$ and $\alpha$ from (105), the integrals in (144) become

$$
\begin{gathered}
\int_{0}^{1} \rho(e+3 \alpha) x^{4} d x=\rho_{0} \sum_{m=0}^{\infty}\left(A_{m}+3 B_{m}\right)\left(\frac{1}{m+5}-\frac{k}{m+7}\right), \\
\int_{0}^{1} \rho x^{4} d x=\rho_{0}\left(\frac{1}{5}-\frac{k}{7}\right) .
\end{gathered}
$$

Convenience in the computations is gained by replacing $A_{m}$ and $B_{m}$ by $C_{m}$ and $D_{m}$ by means of (107) and (108), which give

$$
A_{0}+3 B_{0}=\frac{5}{2} A_{0}, \quad A_{m}+3 B_{m}=\frac{C_{m}+3 D_{m}}{m} \quad(m=2,4, \cdots),
$$

so that (144) reduces to the form

$$
\begin{aligned}
f=A_{0}+\frac{2}{5} \sum_{2}^{\infty} \frac{C_{m}+3 D_{m}}{m}-\frac{14}{5(7-5 k)} \sum_{2}^{\infty} \frac{C_{m}+3 D_{m}}{m+5} & \\
& +\frac{2}{7-5 k} \sum_{2}^{\infty} \frac{C_{m}+3 D_{m}}{m+7} .
\end{aligned}
$$

8. Deflection of apparent gravity. A quantity of importance in the application to the earth is the deflection of apparent gravity relative to the earth's surface due to the action of the disturbing forces.

The equation of the boundary surface of the strained body is

$$
r=a\left(1+e_{1} S_{2}\right),
$$

while that of a level surface may be written

$$
r=a\left(1+\mathrm{e} S_{2}\right) \text {. }
$$


The angle between the normals to the two surfaces (149) and (150) is

$$
\left(\mathrm{e}-e_{1}\right) \frac{\partial S_{2}}{\partial \theta}
$$

The value of e may be found from the condition that the sum of the gravitation potential of the strained body and the disturbing potential is constant at the surface (150).

The change in the external gravitation potential due to the strain may be computed by the same method as that employed in finding the internal potential $U$; it is in fact expressed by the first integral in (35) if the upper limit $r$ is replaced by $a$. Comparing with (140) it is seen that the change in the external potential is equivalent to

$$
\frac{3 \gamma(A-C) S_{2}}{2 r^{3}}=\frac{3 g a^{2} I f S_{2}}{2 r^{3} M}
$$

The external potential of the unstrained body is

$$
\frac{\gamma M}{r}=\frac{g a^{2}}{r}
$$

and the potential of the disturbing forces is

$$
W=\frac{c g}{2 a} r^{2} S_{2}
$$

The sum of these three parts, at the surface (150), to the first order of small quantities, is

$$
g a\left[1+\left(\frac{3 I f}{2 M a^{2}}+\frac{c}{2}-\mathrm{e}\right) S_{2}\right],
$$

and this is constant if

$$
\mathrm{e}=\frac{c}{2}+\frac{3 I f}{2 M a^{2}}
$$

This reduces (151) to

$$
\left(\frac{c}{2}+\frac{3 I f}{2 M a^{2}}-e_{1}\right) \frac{\partial S_{2}}{\partial \theta}
$$

which is the angular deflection of apparent gravity with respect to the boundary surface.

Let $q$ denote the ratio of this quantity to the value it would have if the body were absolutely rigid; then since the latter value is $\frac{1}{2} c \partial S_{2} / \partial \theta$,

$$
q=1+\frac{3 I}{M a^{2}} \cdot \frac{f}{c}-2 \frac{e_{1}}{c}
$$




\section{Application to the earth}

1. Values of constants in case of the earth. In the case of the earth the mean radius and mean density are quite accurately known. Expressed in C. G. S. units the values here used are*

$$
a=6.371 \times 10^{8}, \quad \rho_{m}=5.527, \quad \gamma=6.6576 \times 10^{-8} .
$$

From these result the values

$$
g=982, \quad g \rho_{m} a=3.458 \times 10^{12} .
$$

As regards the variation of density within the earth, it is known that the surface density is about half the mean density, and that the diametral moment of inertia is about $\frac{5}{6}$ of the value for a homogeneous earth of density $\rho_{m}$; i.e.,

$$
\rho_{1}=\frac{1}{2} \rho_{m}, \quad I=\frac{1}{3} M a^{2} .
$$

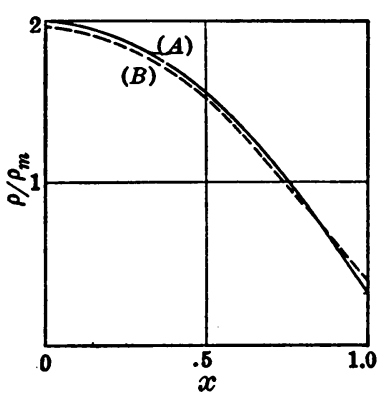

Fra. 1.

These equations can be satisfied only approximately by a density function of two terms, but the approximation can be sufficiently close to serve the present purpose. The formula which has been used is expressed by (94) with $k=\frac{5}{6}$. This gives a close approximation to the correct value of the moment of inertia, but makes the surface density less than that of actual surface rocks. A comparison with the well-known formula known as Laplace's law of density is givent in Table $I$ and in Figure 1.

TABLE I. Comparison of Assumed Law of Density with Laplace's Law

(A) $\rho=\rho_{0}\left(1-k x^{2}\right)=2 \rho_{m}\left(1-\frac{5}{6} x^{2}\right)$,

(B) $\rho=\rho_{0} \frac{\sin \kappa x}{\kappa x}=1.955 \rho_{m} \frac{\sin 2.461 x}{2.461 x}$.

\begin{tabular}{|c|c|c|}
\hline$x=\frac{r}{a}$ & \multicolumn{2}{|c|}{$\rho / \rho_{m}$} \\
\cline { 3 - 3 } & $(A)$ & $(B)$ \\
\hline 0 & 2.00 & 1.95 \\
.2 & 1.93 & 1.87 \\
.4 & 1.73 & 1.65 \\
.6 & 1.40 & 1.31 \\
.8 & .93 & .91 \\
1.0 & .33 & .50 \\
\hline
\end{tabular}

* The values of $\rho_{m}$ and $\gamma$ are those found by C. V. Boys, N a t u r e, vol. 50, p. 419.

$\dagger$ The formula of Laplace satisfies both of equations (159) very closely. The same is true of the trinomial formula

$$
\rho=\rho_{0}\left(1-\frac{14}{13} x^{2}+\frac{21}{65} x^{4}\right),
$$

and numerical solutions using this formula are entirely practicable, but considerable additional labor would be imposed by the addition of a third term to the density formula. 
The values of $\mu$ and $\lambda$ have been determined experimentally for many specimens of surface rocks. Concerning the values throughout the interior of the earth the only evidence is the comparison of the actual yielding of the earth to disturbing forces with the computed yielding. Computations based on the assumption of uniform elasticity throughout the body have made it quite certain that the material of the earth is on the average far more rigid than the surface rocks. The computations which have now been made on the assumption of variable elasticity are based upon formulas (95) with values of the constants $\lambda_{0}, \mu_{0}$, and $h$ so taken that

$$
\lambda_{1}=\mu_{1}=2.542 \times 10^{11} \quad \text { C. G. S. units. }
$$

These surface values are obtained by averaging a considerable number of results given by Adams and Coker.*

2. Assumption regarding $n$. The value of $n$ for the actual tidal disturbance is so small that the terms containing $n$ in the formulas expressing the solution may be neglected in computations relating to the problem of the rigidity of the earth. The problem of forced and free vibrations is, however, of some interest in itself, and one series of numerical results will be given based upon a series of values of $n$.

3. Summary of numerical results for static problem. The numerical results which have been obtained for the static problem fall into three main groups, the design being to show separately the influence of variable density and variable elasticity upon the quantities which are involved in estimates of the rigidity of the earth. The grouping corresponds to the following three assumptions:

(1) $\rho$ and $\mu$ both constant;

(2) $\rho$ variable, $\mu$ constant;

(3) $\rho$ and $\mu$ both variable.

Groups (1) and (2) each consist of two series of results, one for $\lambda=\infty$ (incompressibility), the other for $\lambda=\mu$. The quantities of chief interest are $e_{1} / c, f / c$, and $q$, since it is these whose actual values are inferred from observation; and the results are so arranged as to show the way in which each of these quantities depends upon $\mu$.

* An investigation into the elastic constants of rocks, more especially with reference to cubic compressibility, by Frank D. Adams and Ernest G. Coker, A m e rican J o u rnal of S c i e n c e , vol. 172 (1906), pp. 95-123. Averages of the values found for seventeen specimens of rocks of various kinds are as follows: $\mu=2.542 \times 10^{11}$ dynes $/ \mathrm{cm}^{2}, \lambda+\frac{3}{3} \mu=4.218 \times 10^{11}$ dynes $/ \mathrm{cm}^{2}$. These give $\lambda / \mu=0.993$. 
(1) Case of uniform density and elasticity. (Table II and Figure 2). Although the solution of this case is known, ${ }^{*}$ the series of numerical results here given is more extended than has heretofore been published. Of especial

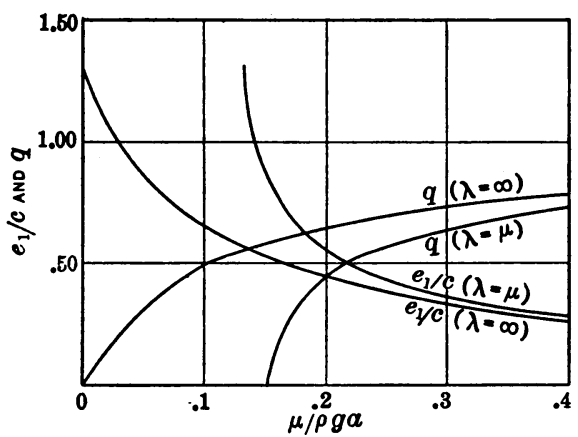

Fig. 2.

interest is the curve representing the results for the case $\lambda=\mu$. It is seen that this curve shows an infinite discontinuity for a value of $\mu / g \rho a$ between 0.12 and 0.125 . This corresponds to the case of "gravitational instability" which is discussed by Love. $\dagger$ Algebraically this case results from the fact

TABLE II. Results for the Case of Uniform Density and Elasticity

\begin{tabular}{|c|c|c|c|c|c|}
\hline \multirow{2}{*}{$\frac{\mu}{\rho g a}$} & \multicolumn{3}{|c|}{$\lambda=\mu$} & \multicolumn{2}{|c|}{$\lambda=\infty$} \\
\hline & $\frac{e_{1}}{c}$ & $\frac{j}{c}$ & $q$ & $\frac{e_{1}}{c}=\frac{f}{c}$ & $q$ \\
\hline 0 & 1.250 & 1.250 & & 1.250 & 0 \\
\hline .05 & .732 & .815 & & .847 & .322 \\
\hline .10 & .247 & .554 & & .641 & .487 \\
\hline .12 & -2.371 & -.030 & & .584 & .533 \\
\hline .125 & 4.955 & 1.472 & & .571 & .543 \\
\hline .15 & .830 & .577 & .032 & .515 & .588 \\
\hline .20 & .535 & .449 & .470 & .431 & .655 \\
\hline .30 & .366 & .331 & .665 & .325 & .740 \\
\hline .40 & .285 & .264 & .747 & .260 & .792 \\
\hline .50 & .235 & .220 & .794 & .217 & .826 \\
\hline
\end{tabular}

* Some Problems of Geodynamics, by A. E. H. Love. See especially Chapters VII and VIII. It is of interest to note the following results given by Love:

$$
\begin{array}{llll}
\frac{\lambda}{\mu}=\frac{46}{25}=1.84, & \frac{\mu}{g \rho a}=\frac{5}{24}=0.208, & \frac{e_{1}}{c}=0.466, & \frac{f}{c}=0.4275 ; \\
\frac{\lambda}{\mu}=\frac{2041}{1944}=1.05, & \frac{\mu}{g \rho a}=0.202, & \frac{e_{1}}{c}=0.522, & \frac{f}{c}=0.436 .
\end{array}
$$

It will be seen that these conform well to the results shown in Fig. 2. In making this comparison it should be nóted that Love's symbols $h, k$ are so defined that

$$
h=2 \frac{e_{1}}{c}, \quad k=\frac{3 I}{M a^{2}} \cdot \frac{f}{c},
$$

so that the quantity above designated by $q$ is, in Love's notation, $1+k-h$.

† Some Problems of Geodynamics, Chapter IX. 
that, for certain values of the elastic constants, equations (120) may be satisfied with $c=0$ (i.e., with zero disturbing force) without the vanishing of the coefficients in the assumed developments of $e$ and $\alpha$.

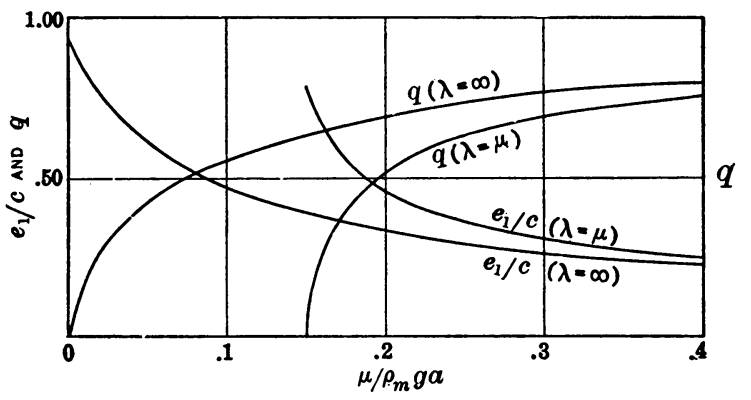

FIG. 3.

(2) Case of variable density but uniform elasticity. (Table III and Figure 3). The effect of the assumption of variable density is seen by comparing the two series of results in Table III and Figure 3 with the corresponding series

TABLE III. Results for the Case of Variable Density and Uniform Elasticity (Values in brackets obtained by extrapolation.)

\begin{tabular}{|c|c|c|c|c|c|c|}
\hline & \multicolumn{3}{|c|}{$\lambda=\mu$} & \multicolumn{3}{c|}{$\lambda=\infty$} \\
\cline { 2 - 7 }$\frac{\rho_{m} g a}{n}$ & $\frac{e_{1}}{c}$ & $\frac{f}{c}$ & $q$ & $\frac{e_{1}}{c}$ & $\frac{f}{c}$ & $q$ \\
\hline 0 & .942 & & & .942 & .910 & 0 \\
.05 & & & & .597 & .643 & .430 \\
.10 & & & & .472 & .514 & .555 \\
.15 & .763 & .532 & .010 & .390 & .426 & .634 \\
.20 & .436 & .395 & .512 & .333 & .365 & .689 \\
.30 & .296 & .291 & .690 & .258 & .283 & .759 \\
.40 & .234 & .236 & .760 & {$[.211]$} & {$[.231]$} & {$[.802]$} \\
\hline
\end{tabular}

in Table II and Figure 2. The variation of the strain with $\mu$ is similar in the two cases, but the strain for a given value of $\mu$ is materially less in the case of variable than in that of uniform density. The two curves for the case $\lambda=\mu$ resemble each other also in the feature of an infinite discontinuity.*

* The numerical solution in the case of variable density involves a considerable amount of labor; it is, however, greatly facilitated by the use of calculating machines. In preparation for the solution of a series of cases with specific values of the elastic moduli, the coefficients in formulas (116), (117), and (118) which depend upon $k$ and $m$ may be computed once for all for values of $m$ as high as may be deemed necessary; these are used in connection with the numerical values of $b$ and of $\lambda / \mu$ appropriate to each specific case. Every $D_{m}$ and $C_{m}$ is thus computed from terms of lower order, the resulting values being linear functions of $C_{2}, D_{2}$, and $A_{0}$. From these the summations occurring in equations (120) may be computed as linear functions of $C_{2}, D_{2}$, and $A_{0}$; it is then easy to complete the solution. 
3. Case in which both density and elasticity are variable. (Table IV and Figure 4). A series of results for the case of variable elasticity was obtained by giving to the constants $\mu_{0}$ and $h$ in the formula $\lambda=\mu=\mu_{0}\left(1-h x^{2}\right)$ four sets of values, so taken that $\mu_{1}$ has always the value $2.542 \times 10^{11}$ already cited as holding for surface rocks, while $\mu_{0} / \mu_{1}$ has the four values $4,5,6 \frac{2}{3}, 10$. The four assumed values of $h$ are $0.75,0.80,0.85,0.90$. Table IV and Figure 4 show the way in which the three quantities $e_{1} / c, f / c$, and $q$ vary with $\mu_{0}$.

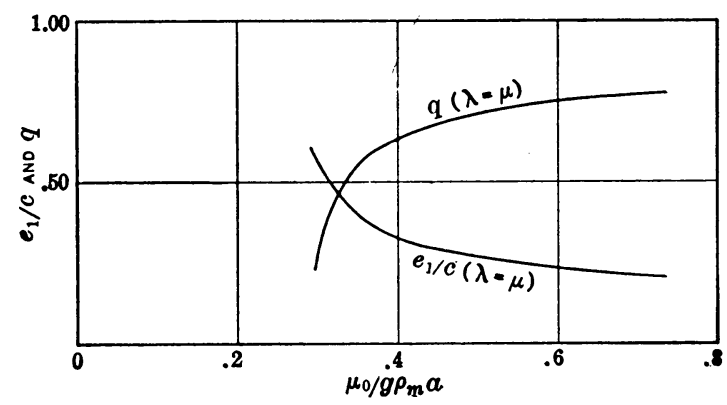

Fig. 4.

4. Rigidity of the earth. Experimental evidence as to the yielding of the earth to small disturbing forces involves the two quantities $f / c$ and $q$.

The value of $f / c$ may be inferred from the earth's free nutation period as determined by observed variations of latitude, the prolongation of the period

TABLE IV. Results for Variable Density and Variable Elasticity

\begin{tabular}{|c|c|c|c|c|c|c|}
\hline$h$ & $\frac{\mu_{0}}{\mu_{1}}$ & $b$ & $\frac{\mu_{0}}{\rho_{m} g a}$ & $\frac{e_{1}}{c}$ & $\frac{f}{c}$ & $q$ \\
\hline .75 & 4 & 3.4025 & .294 & .605 & .440 & .217 \\
.80 & 5 & 2.7220 & .367 & .374 & .335 & .576 \\
.85 & $6 \frac{3}{3}$ & 2.0415 & .490 & .283 & .269 & .695 \\
.90 & 10 & 1.3610 & .735 & .211 & .207 & .779 \\
\hline
\end{tabular}

as compared with that for an absolutely rigid body being accounted for (as Newcomb first pointed out) by elastic yielding.* This line of evidence indicates a value of $f / c$ between 0.27 and 0.29 .

The observed effect of lunar and solar tidal forces upon the direction of gravity relative to the earth at a given place should theoretically give the

* It may be shown that the actual nutation period is to a close approximation the same as that of an unyielding body having the figure which the earth would assume if centrifugal forces were annulled. The theory leading to this result was given by the present writer in a paper presented to the American Mathematical Society, San Francisco Section, Dec. 20, 1902, of which an abstract was published in the Bulletin of the Society, vol. 9 (1903), p. 299. Similar reasoning was employed by J. Larmor in a paper in P r o c e e d in gs of the R o y a l S o ciet y, London, Ser. A, vol. 82 (1909). 
value of $q$. This quantity is, in fact, the ratio of the actual deflection of gravity to the deflection computed on the assumption that the earth is absolutely unyielding. Precise observations of this effect have been made by 0 . Hecker and others by the use of the horizontal pendulum, and by A. A. Michelson by measurement of the actual water tides in closed horizontal pipes. The observations of Hecker* gave values of $q$ varying with azimuth, indicating a greater yielding of the earth in the north-south than in the eastwest azimuth. Michelson's results, $\nmid$ however, indicate equal yielding in all azimuths, the value of $q$ adopted as most probable being 0.69 .

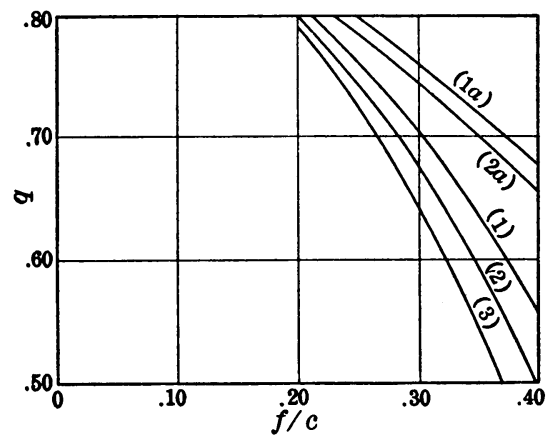

FIG. 5.

The values of $f / c$ and $q$ which are inferred from observation cannot be harmonized with theory on the assumption of homogeneity and incompressibility, and the discrepancy is only partly removed by assuming compressibility. The results above given show that both variable density and variable elasticity work in the direction of harmonizing the computed with the observed values. This is brought out by comparing the simultaneous values of $f / c$ and $q$ in each of the five series of results given in Tables II, III, and IV. The tabulated data are represented graphically in Figure 5, the five curves showing the relation between $f / c$ and $q$ for each of the five series. The curves $(1 a)$ and $(2 a)$ correspond to incompressibility, while (1), (2), and (3) are for the three cases in which $\lambda=\mu$.

Comparing the three curves (1), (2), and (3), it is seen that if $f / c$ lies between

* O. Hecker, Veröffentlichungen des Kgl. Preussischen Institutes, No. 32, Berlin (1907).

$\dagger$ Michelson's preliminary observations are described in a paper entitled Preliminary results of measurements of the rigidity of the earth, Journal of $\mathrm{Geology}$, vol. 22 (1914). See also Astrophysical Journal, vol. 39 (1914). These measurements gave 0.71 as the best value of $q$. (For a correction to the results originally published see S ci e n c e, October 3,1919, p. 327.) The value 0.69 was obtained from a more extended series of measurements, the aceount of which has not at this date (October, 1919) been published. The writer is indebted to Professor Michelson and Professor Henry G. Gale for the communication of the general result in advance of publication. 
0.27 and 0.29 (as indicated by latitude observations), $q$ should fall within the limits

0.74 and 0.72 in case (1), 0.72 and 0.69 in case (2), 0.69 and 0.66 in case (3).

Materially greater values of $q$ are indicated by the curves $(1 a)$ and $(2 a)$.

Considering all the computations that have been made, the best agreement with the facts of observation results from the assumptions

$$
\rho=\rho_{0}\left(1-\frac{5}{6} x^{2}\right), \quad \lambda=\mu=\mu_{0}\left(1-.85 x^{2}\right),
$$

the value of $\rho_{0}$ being based upon the known value of $\rho_{m}$, and that of $\mu_{0}$ being such as to make $\mu_{1}$ agree with the value found experimentally for surface rocks. This surface value is

$$
\mu_{1}=2.542 \times 10^{11} \text { dynes } / \mathrm{cm}^{2},
$$

giving for the value at the center

$$
\mu_{0}=\mu_{1} / .15=1.695 \times 10^{12} \mathrm{dynes} / \mathrm{cm}^{2} .
$$

5. Forced oscillation. The numerical results given above are for the static problem, all terms in the formulas which contain $n$ being regarded as negligible. Inspection of equations (118) shows, however, that the same routine of computation can be followed when $n$ has any known value. It is thus possible to determine the forced oscillation due to a disturbing potential of any known period.

6. Free oscillation. If an oscillation of the type assumed in the solution is possible in the absence of disturbing forces, equations (120) must be satisfied with $c=0$. Obviously this is possible only for particular values of $n$, and the only method which suggests itself of determining such particular values is by interpolation in a series of results computed with assumed values of $n$. This method, though somewhat laborious, is entirely feasible, and has actually been carried out in one case with results which will be given.

If we consider a continuous series of forced oscillations with the same intensity of disturbing force but with period decreasing from $\infty$ (corresponding to static strain), the period of possible free oscillation will be that at which the forced oscillation reverses its phase while its amplitude passes through $\infty$. If, therefore, for each of a series of values of $n$, beginning with 0 , the values of $C_{2} / c, D_{2} / c, A_{0} / c$ are computed, each of these will increase in magnitude with increasing $n$, approaching positive or negative infinity as $n$ approaches the value corresponding to the free oscillation, then changing sign and decreasing in magnitude. The required critical value of $n$ may be found by interpolation in one of these series, preferably by taking reciprocals and interpolating for the zero-value. 
The case for which computations have been made is that in which the density and elastic moduli are represented by equations (94) and (95) with $k=\frac{5}{6}$ and $h=0.85$. Results for $n=0$ have already been given in Table IV, and further computations have been made for $n=1,2$, and 3 , giving the results shown in Table V.

TABLE V. Results for Case of Forced Oscillation

[Results in brackets obtained by interpolation for case of free vibration.]

\begin{tabular}{|c|c|c|c|c|c|c|}
\hline$n$ & $\begin{array}{l}\text { Peri } \\
\text { seco }\end{array}$ & $\frac{C_{2}}{c}$ & $\frac{D_{2}}{c}$ & $\frac{A_{0}}{c}$ & $\frac{e_{1}}{c}$ & $\frac{c}{e_{1}}$ \\
\hline $\left.\begin{array}{c}0 \\
1 \\
2 \\
{\left[\begin{array}{c}2.77 \\
3\end{array}\right]}\end{array}\right]$ & $\begin{array}{c}\infty \\
5060 \\
3578 \\
{[3040]} \\
2922\end{array}$ & $\begin{array}{c}.795 \\
1.668 \\
6.820 \\
{[ \pm \infty]} \\
-44.04\end{array}$ & $\begin{array}{l}-.540 \\
-1.146 \\
-4.687 \\
{[ \pm \infty]} \\
+30.90\end{array}$ & $\begin{array}{c}.381 \\
.608 \\
1.492 \\
{[ \pm \infty} \\
-5.857\end{array}$ & {$\left[\begin{array}{c}.436 \\
.948 \\
\pm \infty \infty \\
-2.93\end{array}\right]$} & $\begin{array}{r}2.29 \\
1.06 \\
{[0]} \\
-.34\end{array}$ \\
\hline
\end{tabular}

The interpolation for the critical value of $n$ may be accomplished to a good approximation by means of the graph $(A)$ shown in Figure 6, which represents $c / e_{1}$ as a function of $n$; the zero-value falling at about $n=2.77$.

From the meaning of $p$ as given by (27), it is obvious that the period of a complete oscillation is $2 \pi / p$ or $2 \pi \sqrt{a / n g}$. Putting $a=6.371 \times 10^{8} \mathrm{~cm}$., $g=982 \mathrm{~cm} / \mathrm{sec}^{2}$, the period in seconds is $5060 / \sqrt{n}$. The period corresponding to $n=2.77$ is thus about 3040 seconds.

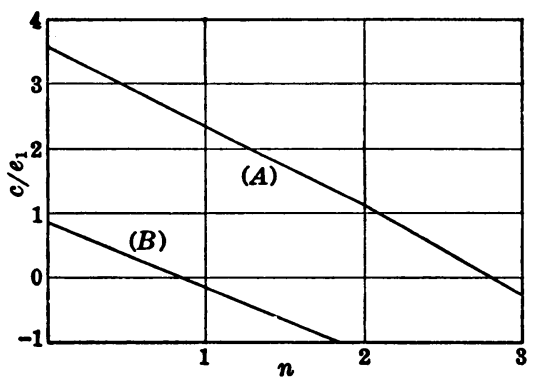

Fig. 6.

It is of interest to compare this result with that given by equation (138) for a homogeneous incompressible fluid sphere. The critical value of $n$ for this case is 0.8 , corresponding to a free oscillation period of 5650 seconds. The graph of $c / e_{1}$ as a function of $n$ is the straight line $(B)$ shown in Figure 6. 